\title{
Application of renormalized coupled-cluster methods to potential function of water
}

\author{
Piotr Piecuch • Marta Włoch • António J. C. Varandas
}

Received: 20 November 2006 / Accepted: 10 March 2007 / Published online: 15 May 2007

(C) Springer-Verlag 2007

\begin{abstract}
The goal of this paper is to examine the performance of the conventional and renormalized singlereference coupled-cluster (CC) methods in calculations of the potential energy surface of the water molecule. A comparison with the results of the internally contracted multireference configuration interaction calculations including the quasi-degenerate Davidson correction $(\mathrm{MRCI}(\mathrm{Q}))$ and the spectroscopically accurate potential energy surface of water resulting from the use of the energy switching (ES) approach indicates that the relatively inexpensive completely renormalized (CR) CC methods with singles (S), doubles (D), and a non-iterative treatment of triples (T) or triples and quadruples (TQ), such as CR-CCSD(T), CR-CCSD(TQ), and the recently developed rigorously size extensive extension of $\mathrm{CR}-\mathrm{CCSD}(\mathrm{T})$, termed CR-CC $(2,3)$, provide substantial improvements in the results of conventional $\operatorname{CCSD}(\mathrm{T})$ and CCSD(TQ) calculations at larger internuclear separations. It is shown that the $\mathrm{CR}-\mathrm{CC}(2,3)$ results corrected for the effect of quadruply excited clusters through the CR-CC(2,3)+Q approach can compete with the highly accurate $\operatorname{MRCI}(\mathrm{Q})$ data. The excellent agreement between the $\mathrm{CR}-\mathrm{CC}(2,3)+\mathrm{Q}$ and $\mathrm{MRCI}(\mathrm{Q})$ results suggests ways of improving the global
\end{abstract}

Contribution to the Mark S. Gordon 65th Birthday Festschrift Issue.

P. Piecuch · M. Włoch

Department of Chemistry, Michigan State University,

East Lansing, MI 48824, USA

P. Piecuch $(\varangle)$

Department of Physics and Astronomy, Michigan State University,

East Lansing, MI 48824, USA

e-mail: piecuch@chemistry.msu.edu

P. Piecuch · A. J. C. Varandas

Departamento de Química, Universidade de Coimbra,

3004-535 Coimbra, Portugal potential energy surface of water resulting from the use of the ES approach in the regions of intermediate bond stretches and intermediate energies connecting the region of the global minimum with the asymptotic regions.

Keywords Coupled-cluster theory $\cdot$ Renormalized coupled-cluster methods · Potential energy surfaces . Potential function of water

\section{Introduction}

The key to a successful description of molecular potential energy surfaces involving bond making and breaking is an accurate and balanced treatment of dynamical and non-dynamical electron correlation effects. Balancing dynamical and non-dynamical correlation effects in molecular systems in a proper manner has been one of the main themes of the research conducted by Professor Mark S. Gordon, as reflected, for example, in a large number of truly inspiring computational studies of reaction pathways and mechanisms in various areas of chemistry, carried out over the years by Professor Mark S. Gordon and co-workers. In this paper, we focus on the problem of balancing dynamical and nondynamical correlations in coupled-cluster (CC) theory [1-5] (cf. Refs. [6-14] for selected reviews).

Conventional single-reference CC methods, such as, for example, the popular $\operatorname{CCSD}(\mathrm{T})$ approach [15], in which a non-iterative correction due to triply excited clusters (T) is added to the CCSD (CC singles and doubles) [16-18] energy, and its extensions to quadruply excited clusters (Q) through the $\operatorname{CCSD}\left(\mathrm{TQ}_{\mathrm{f}}\right)$ [19], $\operatorname{CCSD}(\mathrm{TQ}), \mathrm{b}$ [20], and similar schemes, provide an excellent description of dynamical correlation effects, which dominate electron correlations in the 
closed-shell regions of potential energy surfaces, but they completely fail when the bond breaking, biradicals, and other situations characterized by larger non-dynamical correlation effects are studied (see, e.g., Refs. [7,10-14,20-57] for representative examples). Traditionally, the adequate treatment of ground- and excited-state potential energy surfaces along bond breaking coordinates and other cases of electronic quasi-degeneracies has been the domain of expert multi-reference methods, and a great deal of progress has been achieved in the area of multi-reference calculations, but even the most successful multi-reference approaches are not without limitations. For example, the low-order multireference perturbation theory (MRPT) methods (cf., e.g., Refs. [58,59], and references therein), such as the popular second-order CASPT2 approach [60-63], may encounter serious difficulties with balancing dynamical and nondynamical correlations in studies of chemical reaction pathways and relative energetics of systems characterized by a varying degree of biradical character $[49,50,64]$, while the more robust multi-reference configuration interaction (MRCI) approaches, including the highly successful internally contracted MRCI approach with quasi-degenerate Davidson corrections (the MRCI $(\mathrm{Q})$ method $[65,66])$, are often prohibitively expensive. One should also keep in mind that all multi-reference theories require that the user specifies several additional parameters that do not enter singlereference calculations, such as active orbitals or multiple reference determinants and, particularly in the case of MRCI, numerical thresholds for neglecting unimportant electron configurations. At present, a universally applicable and accepted methodology for choosing these parameters is absent $[67,68]$, and a great deal of expertise and experience is required to perform multi-reference calculations in a proper manner (cf., e.g., Ref. [58]).

From this point of view, the development of practical single-reference procedures that could be applied to at least some of the most frequent multi-reference situations, such as single and double bond dissociations, biradicals, and excited states dominated by two-electron transitions, and that could provide a balanced description of dynamical and nondynamical correlation effects with a more or less black-box effort would be an important step toward widespread progress. This is not to diminish the role of multi-reference approaches, which clearly have the much desired flexibility needed for the accurate treatment of all kinds of electronic quasi-degeneracies. We are only reflecting on the fact that single-reference methods are computational black-boxes, which are generally much easier to use when compared to multi-reference approaches and which have significantly contributed to the popularity of the computational quantum chemistry techniques. Thus, it is useful to explore the possibility of attempting to solve at least some classes of chemical problems involving larger non-dynamical correlation effects without resorting to multi-reference calculations. This has been the motivation behind the recent development of the non-iterative $\mathrm{CC}$ approaches based on the partitioning of the similarity-transformed Hamiltonian pursued by Head-Gordon and co-workers [52-56] (cf., also, Ref. [69]), adopted in a slightly modified form by Hirata et al. $[38,70]$ and commonly labeled as the $\operatorname{CCSD}(2)$ approximations, the spin-flip CC methods developed by Krylov and co-workers [71-73], the iterative and non-iterative methods [12,13,39, 55,56,74-76] based on the extended CC theories of Arponen and Bishop [77-83] and Piecuch and Bartlett [84], and the renormalized and other $\mathrm{CC}$ approaches based on the method of moments of CC equations [10-14,20,29,30,35,36,39$42,44,76,85-88]$, including those that have been incorporated (cf., e.g., Refs. [41,87,89]) in the GAMESS package [90], maintained and distributed at no cost by Professor Mark S. Gordon and Dr. Michael W. Schmidt.

In this work, we focus on the renormalized CC methods for ground electronic states [10-14,20,29,30,39-42,44], which represent a new generation of non-iterative single-reference $\mathrm{CC}$ approaches that are designed to improve the results of the $\operatorname{CCSD}(\mathrm{T})$ and $\operatorname{CCSD}(\mathrm{TQ})$ calculations in the bond breaking/biradical regions of molecular potential energy surfaces, while preserving the ease-of-use (black-box character) and the relatively low computer costs of the $\operatorname{CCSD}(\mathrm{T})$ and CCSD(TQ) approximations. In addition to the original and well-established CR-CCSD(T) (completely renormalized $\operatorname{CCSD}(\mathrm{T})$ ) method $[10-13,20,29,30]$, in which a simple noniterative correction due to triply excited clusters is added to the CCSD energy, and its CR-CCSD(TQ) (completely renormalized CCSD(TQ)) extensions abbreviated as CR-CCSD(TQ), a and CR-CCSD(TQ),b, and described in Refs. [10-13,20,29,30] as well, in which corrections due to triply and quadruply excited clusters are added to the CCSD energy, we examine the performance of the recently developed rigorously size extensive generalization of CR-CCSD(T), termed CR-CC(2,3), and its augmented CR-CC $(2,3)+Q(a)$ and $C R-C C(2,3)+Q(b)$ versions. The CR-CC $(2,3)$ approach, in which the CCSD energy is corrected for triples in a manner similar to the $\operatorname{CCSD}(\mathrm{T})$, CR-CCSD (T), and other non-iterative triples approximations, has been described in detail in Refs. [41,42,44]. In the $\mathrm{CR}-\mathrm{CC}(2,3)+\mathrm{Q}(\mathrm{a})$ and $\mathrm{CR}-\mathrm{CC}(2,3)+\mathrm{Q}(\mathrm{b})$ methods, the $\mathrm{CR}-\mathrm{CC}(2,3)$ energies are approximately corrected for the dominant quadruples effects, estimated by forming the differences of the CR-CCSD(TQ), a (the CR-CC $(2,3)+Q$ (a) case) or CR-CCSD(TQ),b (the CR-CC $(2,3)+\mathrm{Q}(\mathrm{b})$ case) and CR-CCSD(T) energies. Thus, the CR-CC $(2,3)+\mathrm{Q}(x), x=$ $\mathrm{a}, \mathrm{b}$, energies are calculated as $\mathrm{CR}-\mathrm{CC}(2,3)+\mathrm{Q}(x)=$ CR-CC $(2,3)+[$ CR-CCSD(TQ), $x-$ CR-CCSD(T)] (cf., e.g., Refs. [49,50]). We consider two variants of the CR-CC (2,3) approach, namely, the full CR-CC $(2,3)$ method, as described in Refs. [41,42,44], and the approximate 
CR-CC(2,3),A variant, in which the diagonal matrix elements of the similarity-transformed Hamiltonian of CCSD involving triply excited determinants, $\left\langle\Phi_{i j k}^{a b c}\left|\bar{H}^{(\mathrm{CCSD})}\right|\right.$ $\left.\Phi_{i j k}^{a b c}\right\rangle$, which enter the $\mathrm{CR}-\mathrm{CC}(2,3)$ triples correction, are replaced by the spin-orbital energy differences characterizing triple excitations, $\left(\epsilon_{a}+\epsilon_{b}+\epsilon_{c}-\epsilon_{i}-\epsilon_{j}-\epsilon_{k}\right)$, where $\epsilon_{p}$ are the usual spin-orbital energies (see, e.g., Ref. [44]). It is interesting to examine the full implementation of $\mathrm{CR}-\mathrm{CC}(2,3)$ as well as its approximate $\mathrm{CR}-\mathrm{CC}(2,3), \mathrm{A}$ version, since the $\mathrm{CR}-\mathrm{CC}(2,3)$,A approach is equivalent to the $\operatorname{CCSD}(2)_{T}$ method of Ref. [38], which also aims at eliminating or reducing the failures of $\operatorname{CCSD}(\mathrm{T})$ at larger internuclear separations (see Refs. $[41,42,44]$ for further details). The complete list of all CC methods used in this work, papers in which these methods were introduced and described, and scalings of the most expensive CPU steps characterizing each CC method employed in this study with the numbers of occupied and unoccupied orbitals in a molecular basis set used in correlated calculations, $n_{o}$ and $n_{u}$, respectively, are summarized in Table 1.

It has been demonstrated that the $\mathrm{CR}-\mathrm{CCSD}(\mathrm{T})$ and CR-CC $(2,3)$ approaches (particularly, CR-CC $(2,3))$ provide very good results for single bond breaking [10-13,29-31, $33,34,37,40-44,89,91,92]$, and reaction pathways involving biradicals and similar cases of electronic quasi-degeneracies $[42,46-51,59,93,94]$, eliminating the failures of the conven- tional $\operatorname{CCSD}(\mathrm{T})$ and $\operatorname{CCSD}(\mathrm{TQ})$ methods in those multireference situations. The $\mathrm{CR}-\mathrm{CCSD}(\mathrm{TQ}), \mathrm{a}$ and $\mathrm{CR}$ CCSD(TQ),b extensions of CR-CCSD(T) provide further improvements in the results for single bond breaking [11$13,29,31,33]$, while helping to obtain reasonable accuracies in cases of multiple bond stretching or breaking [11-13,20, 28-30,36,39-41,44,76] (cf., also, Ref. [57] for the analogous findings for the approximate versions of CR-CCSD(TQ)). However, with an exception of one study of a global potential energy surface of the BeFH system [37], none of the previous calculations have examined the performance of the CR-CC approaches in large-basis-set calculations for different potential energy surface cuts of a triatomic molecule. This is particularly true for the recently developed $[41,42,44] \mathrm{CR}$ $\mathrm{CC}(2,3)$ approach. According to the benchmark calculations reported in Refs. [41,42,44] and several applications to reaction mechanisms in organic $[42,51]$ and bioinorganic $[49,50]$ chemistries, the $\mathrm{CR}-\mathrm{CC}(2,3)$ approach provides the results which are competitive or at least as good as those obtained with $\operatorname{CCSD}(\mathrm{T})$ for closed-shell molecules near the equilibrium geometries, while improving the already reasonable results of the CR-CCSD(T) calculations in the biradical/bond breaking regions. Moreover, the $\mathrm{CR}-\mathrm{CC}(2,3)$ approach appears to be substantially more accurate than another type of renormalized CC approach, termed LR-CCSD(T) [40,95], and the aforementioned $\operatorname{CCSD}(2)_{T}[38]$ method $[41,42,44]$.
Table 1 The list of CC methods used in this work, papers in which these methods were introduced, and scalings of the most expensive CPU steps characterizing each method with the numbers of occupied $\left(n_{o}\right)$ and unoccupied $\left(n_{u}\right)$ orbitals in a molecular basis set used in correlated calculations

\begin{tabular}{llll}
\hline Method & Reference(s) & \multicolumn{2}{c}{ The most expensive CPU steps } \\
\cline { 2 - 3 } & & Iterative & Non-iterative \\
\hline $\operatorname{CCSD}^{\mathrm{a}}$ & {$[16-18]$} & $n_{o}^{2} n_{u}^{4}$ & $n_{o}^{2} n_{u}^{4}$ \\
$\mathrm{CCSD}(\mathrm{T})$ & {$[15]$} & $n_{o}^{2} n_{u}^{4}$ & $n_{o}^{3} n_{u}^{4}$ \\
$\mathrm{CR}-\mathrm{CCSD}(\mathrm{T})$ & {$[10,30]$} & $n_{u}^{2} n_{u}^{4}$ & $2 n_{o}^{3} n_{u}^{4}$ \\
$\mathrm{CR}-\mathrm{CC}(2,3), \mathrm{A} \equiv \mathrm{CCSD}(2)_{T}{ }^{\mathrm{b}}$ & {$[38,41,42,44]$} & $n_{o}^{2} n_{u}^{4}$ & $2 n_{o}^{3} n_{u}^{4}$ \\
$\mathrm{CR}-\mathrm{CC}(2,3)^{\mathrm{c}}$ & {$[41,42,44]$} & $n_{o}^{2} n_{u}^{4}$ & $2 n_{o}^{3} n_{u}^{4}$ \\
$\mathrm{CCSD}(\mathrm{TQ}), \mathrm{b}^{\mathrm{d}}$ & {$[20]$} & $n_{o}^{2} n_{u}^{4}$ & $n_{o}^{3} n_{u}^{4}(\mathrm{~T}), n_{o}^{2} n_{u}^{5}(\mathrm{Q})$ \\
$\mathrm{CR}-\mathrm{CCSD}(\mathrm{TQ}), x(x=\mathrm{a}, \mathrm{b})^{\mathrm{e}}$ & {$[20]$} & $n_{o}^{2} n_{u}^{4}$ & $2 n_{o}^{3} n_{u}^{4}(\mathrm{~T}), 2 n_{o}^{2} n_{u}^{5}(\mathrm{Q})$ \\
$\mathrm{CR}-\mathrm{CC}(2,3)+\mathrm{Q}(x)(x=\mathrm{a}, \mathrm{b})^{\mathrm{f}}$ & & $2 n_{o}^{3} n_{u}^{4}(\mathrm{~T}), 2 n_{o}^{2} n_{u}^{5}(\mathrm{Q})$ \\
\hline
\end{tabular}

${ }^{a}$ Reference [16] describes the spin-orbital formulation of CCSD. For the non-orthogonally spin-adapted formulation of CCSD for singlet states, see Ref. [17]. For the orthogonally spin-adapted CCSD theory for singlet states, see Ref. [18]

${ }^{\mathrm{b}} \operatorname{CCSD}(2)_{T}$ is obtained by neglecting contributions due to connected quadruply excited clusters in the CCSD(2) method of Ref. [70]. It can also be obtained from the full CR-CC $(2,3)$ approach of Refs. [41,42] by replacing the diagonal matrix elements of the similarity-transformed Hamiltonian of CCSD involving triply excited determinants, $\left\langle\Phi_{i j k}^{a b c}\left|\bar{H}^{(\mathrm{CCSD})}\right| \Phi_{i j k}^{a b c}\right\rangle$, which enter the CR-CC $(2,3)$ triples correction, by the spin-orbital energy differences characterizing triple excitations, $\left(\epsilon_{a}+\epsilon_{b}+\epsilon_{c}-\epsilon_{i}-\epsilon_{j}-\epsilon_{k}\right)$, where $\epsilon_{p}$ are the usual spin-orbital energies [44]

${ }^{c}$ The full CR-CC $(2,3)$ approach, also referred to in Refs. $[41,44]$ as variant D of the CR-CC $(2,3)$ or CR-CCSD $(\mathrm{T})_{\mathcal{L}}$ method

${ }^{\mathrm{d}} \mathrm{CCSD}(\mathrm{TQ}), \mathrm{b}$ can be obtained from the factorized $\operatorname{CCSD}\left(\mathrm{TQ}_{\mathrm{f}}\right)$ approach of Ref. [19] by replacing the first-order estimate for the doubly excited $T_{2}$ clusters in the correction due to quadruples defining the $\operatorname{CSSD}\left(\mathrm{TQ}_{\mathrm{f}}\right)$ energy by the CCSD value of $T_{2}$

${ }^{\mathrm{e}} \mathrm{CR}-\mathrm{CCSD}(\mathrm{TQ})$, a is equivalent to the CR-CCSD(TQ) approach defined in Refs. [10,30]. CR-CCSD(TQ), b is obtained from CR-CCSD(TQ), a by replacing the first-order estimate for the doubly excited $T_{2}$ clusters in the correction due to quadruples defining the CR-CCSD(TQ),a energy by the CCSD value of $T_{2}$

${ }^{\mathrm{f}} \mathrm{CR}-\mathrm{CC}(2,3)+\mathrm{Q}(x)=\mathrm{CR}-\mathrm{CC}(2,3)+[\mathrm{CR}-\mathrm{CCSD}(\mathrm{TQ}), x-\mathrm{CR}-\mathrm{CCSD}(\mathrm{T})], x=\mathrm{a}, \mathrm{b}$ 
As shown in Refs. [41,42,44], the CR-CC $(2,3)$ theory seems to provide the results of the full CCSDT (CC singles, doubles, and triples) $[96,97]$ quality when bond breaking is examined. It is, therefore, very interesting to investigate how the CR-CC $(2,3)$ approach performs when a few different potential energy surface cuts of a triatomic molecule are examined. As explained in Refs. [41,42], the CR-CC $(2,3)$ approach can be extended to quadruple excitations through the CR$\mathrm{CC}(2,4)$ theory, but the CR-CC $(2,4)$ method has not been implemented yet and we may have to investigate formal issues such as the coupling of triples and quadruples in the definition of the CR-CC $(2,4)$ energy corrections, ignored in the original papers on the CR-CC $\left(m_{A}, m_{B}\right)$ methods [41,42]. On the other hand, a highly accurate description of potential energy surfaces that might help various spectroscopic and dynamical studies may require the inclusion of quadruples, in addition to triples that are already well described by the CR-CC $(2,3)$ theory. Thus, in this paper we examine the effect of quadruples on the CR-CC $(2,3)$ results by adding the a posteriori corrections due to quadruply excited clusters extracted, as explained above, from the CR-CCSD(TQ), a or CR-CCSD(TQ),b calculations, to the CR-CC $(2,3)$ energies.

The CR-CCSD(T), CR-CCSD(TQ),a, CR-CCSD(TQ),b, $\mathrm{CR}-\mathrm{CC}(2,3), \mathrm{A} \equiv \mathrm{CCSD}(2)_{T}, \mathrm{CR}-\mathrm{CC}(2,3), \mathrm{CR}-\mathrm{CC}(2,3)$ $+\mathrm{Q}(\mathrm{a})$, and $\mathrm{CR}-\mathrm{CC}(2,3)+\mathrm{Q}(\mathrm{b})$ methods, and their conventional (i.e., not renormalized) CCSD(T) and CCSD(TQ),b ana$\operatorname{logs}$ are carefully tested by examining three important cuts of the global potential energy surface of the water molecule. Those cuts are: (i) the dissociation of a single $\mathrm{O}-\mathrm{H}$ bond which correlates with the $\mathrm{H}\left(1 s^{2} S\right)+\mathrm{OH}\left(X^{2} \Pi\right)$ asymptote, (ii) the simultaneous dissociation of both $\mathrm{O}-\mathrm{H}$ bonds of the water molecule correlating with the $2 \mathrm{H}\left(1 s^{2} S\right)+$ $\mathrm{O}\left(2 p^{4}{ }^{3} P\right)$ channel, and (iii) the $C_{2 v}$ dissociation pathway of the water molecule into $\mathrm{H}_{2}\left(X^{1} \Sigma_{g}^{+}\right)$and $\mathrm{O}\left(2 p^{4}{ }^{1} D\right)$. The CR-CCSD(T), CR-CCSD(TQ), a, CR-CCSD(TQ),b, CR-CC (2,3),A， CR-CC(2,3), CR-CC(2,3)+Q(a), and CR-CC $(2,3)+Q(b)$ results and the corresponding CCSD, $\operatorname{CCSD}(\mathrm{T})$, and $\operatorname{CCSD}(\mathrm{TQ}), \mathrm{b}$ results, all obtained with the basis sets of the aug-cc-pCVXZ $(X=\mathrm{D}, \mathrm{T}, \mathrm{Q})$ quality [98101], are compared with the results of the large-scale $\operatorname{MRCI}(\mathrm{Q})$ calculations, also carried out in this work, and the spectroscopically accurate global potential energy surface of water resulting from the use of the energy switching (ES) approach [102].

We chose the water molecule as our benchmark system for a number of reasons. Clearly, water is among the most important molecules and a prototype system for a variety of spectroscopic and reaction dynamics studies. There are many applications involving water molecule in which the knowledge of a reliable potential energy surface is required. Selected examples of such applications include the spectrum of the water vapor, which is important for the understanding of the absorption and retention of sunlight in Earth's atmos- phere and physics of other planets and stars [103-112], and combustion studies involving hot steam. For example, the $\mathrm{O}\left(2 p^{4}{ }^{1} D\right)+\mathrm{H}_{2}\left(X^{1} \Sigma_{g}^{+}\right) \rightarrow \mathrm{OH}\left(X^{2} \Pi\right)+\mathrm{H}\left(1 s^{2} S\right)$ reaction, which takes place on the ground-state potential energy surface of water, is known to play a significant role in combustion and atmospheric chemistry $[113,114]$. Two of the dissociation pathways examined in this work, namely, the dissociation of a single $\mathrm{O}-\mathrm{H}$ bond and the $C_{2 v}$ dissociation path of the water molecule into $\mathrm{H}_{2}\left(X^{1} \Sigma_{g}^{+}\right)$and $\mathrm{O}\left(2 p^{4}{ }^{1} D\right)$ are directly related to this important reaction. The water molecule has received considerable attention in recent years due to several attempts to produce the spectroscopically and dynamically accurate global potential energy surface using $a b$ initio and other theoretical means [102,109,115-119]. One such attempt has resulted in the ES potential function used in this work [102]. By comparing various CR-CC and $\mathrm{MRCI}(\mathrm{Q})$ data with the energies provided by the ES potential function, we may suggest ways of improving the ES and similar potentials in intermediate-energy regions where no precise or well understood spectroscopic or ab initio data are available. Thus, in addition to testing CR-CC methods, we may contribute to the ongoing effort to produce the high accuracy water surface which could be used in a variety of spectroscopic and dynamical applications.

\section{Computational details}

In order to illustrate the performance of the renormalized $\mathrm{CC}$ methods discussed in the Introduction and listed in Table 1, we performed the CR-CCSD(T), CR-CCSD(TQ),a, CR-CCSD(TQ),b, CR-CC(2,3),A $\quad\left(=\operatorname{CCSD}(2)_{T}\right)$, CR-CC(2,3), CR-CC(2,3)+Q(a), and CR-CC (2,3)+Q(b) calculations for the three cuts of the global potential energy surface of the water molecule. Those cuts are: (i) the dissociation of a single $\mathrm{O}-\mathrm{H}$ bond, which correlates with the $\mathrm{H}\left(1 s^{2} S\right)+\mathrm{OH}\left(X^{2} \Pi\right)$ asymptote, (ii) the simultaneous dissociation of both $\mathrm{O}-\mathrm{H}$ bonds, which correlates with the $2 \mathrm{H}\left(1 s^{2} S\right)+\mathrm{O}\left(2 p^{4}{ }^{3} P\right)$ channel, and (iii) the $C_{2 v}$ dissociation pathway of the water molecule into $\mathrm{H}_{2}\left(X^{1} \Sigma_{g}^{+}\right)$and $\mathrm{O}\left(2 p^{4}{ }^{1} D\right)$. In the case of cut (i), one of the two $\mathrm{O}-\mathrm{H}$ bonds and the $\mathrm{H}-\mathrm{O}-\mathrm{H}$ angle were kept fixed at their respective equilibrium values taken from Ref. [109] $\left(R_{e}=0.95785 \AA\right.$ and $\alpha_{e}=104.501$ degree, respectively). In the case of the $C_{2 v^{-}}$ symmetric cut (ii), the $\mathrm{H}-\mathrm{O}-\mathrm{H}$ angle $\alpha$ was kept fixed at its equilibrium value taken from Ref. [109] $\left(\alpha_{e}=104.501\right.$ degree). In the case of another $C_{2 v}$-symmetric cut, namely cut (iii), we followed the approximate energy path toward the dissociation of the water molecule into $\mathrm{H}_{2}\left(X^{1} \Sigma_{g}^{+}\right)$and $\mathrm{O}\left(2 p^{4}{ }^{1} D\right)$, determined using the potential function of Ref. [102] and defined by the coordinate $Y$, which is the distance between the $\mathrm{O}$ nucleus and the line connecting the $\mathrm{H}$ nuclei, and the properly optimized $\mathrm{H}-\mathrm{O}-\mathrm{H}$ angle $\alpha[113,114]$. The equilibrium values of $Y$ and $\alpha$ are $Y_{e}=0.58641 \AA$ and 
$\alpha_{e}=104.501$ degree. The results of various CR-CC calculations are compared with those obtained with the conventional CCSD, CCSD(T), and CCSD(TQ),b methods, which are very well suited for the equilibrium region but are expected to face considerable difficulties when water starts to dissociate into open-shell fragments, and with the results obtained with the MRCI $(\mathrm{Q})$ approach, which is capable of providing an accurate global potential energy surface, including all three dissociation channels listed above.

We also compare the results of various CR-CC and $\operatorname{MRCI}(\mathrm{Q})$ calculations with the highly accurate global potential energy surface of water resulting from the use of the ES approach of Varandas [102]. The ES surface of Ref. [102] was obtained by combining and further refining the manybody expansion [120] potential of Murrell and Carter [115] and the polynomial potential form of Polyansky, Jensen, and Tennyson [116]. The ES surface has a spectroscopic or nearly spectroscopic $\left(\sim 1-10 \mathrm{~cm}^{-1}\right)$ accuracy up to about 19,000 $\mathrm{cm}^{-1}$ above the global minimum and remains quite accurate at higher energies. With the proper treatment of long-range forces and other suitable refinements, the ES potential of Ref. [102] has an overall double many-body expansion [121124] quality, making it very useful to study reaction dynamics involving water. In particular, the ES potential of Ref. [102] used in this work, and its multi-sheeted extensions [118, 119], have been exploited in a number of dynamical calculations, including, for example, the successful rate constant and cross-section calculations for the $\mathrm{O}\left(2 p^{4}{ }^{1} D\right)+\mathrm{H}_{2}\left(X^{1} \Sigma_{g}^{+}\right)$ reaction $[113,114]$. As mentioned in the Introduction, the $\mathrm{O}\left(2 p^{4}{ }^{1} D\right)+\mathrm{H}_{2}\left(X^{1} \Sigma_{g}^{+}\right) \rightarrow \mathrm{OH}\left(X^{2} \Pi\right)+\mathrm{H}\left(1 s^{2} S\right)$ reaction, which takes place on the ground-state potential energy surface of water, is known to play a significant role in combustion and atmospheric chemistry. Two of the above cuts (cut (i) and (iii)) are directly related to this process. In addition to testing the CR-CC (also, $\mathrm{MRCI}(\mathrm{Q}))$ methods, by comparing the best CR-CC and MRCI(Q) data with the energies provided by the ES potential function, we suggest ways of improving the ES potential, particularly in intermediate and selected higher-energy regions where precise or well understood spectroscopic data are not always available and where, as shown in this work, the best CR-CC and MRCI(Q) calculations almost perfectly agree with each other.

All CC and CR-CC calculations were performed using the spin- and symmetry-adapted restricted Hartree-Fock (RHF) determinant as a reference. The $\operatorname{MRCI}(\mathrm{Q})$ calculations were performed using the usual multi-determinantal reference obtained in the single-root complete-active-space selfconsistent-field (CASSCF) calculations. The active space used in the CASSCF and subsequent MRCI(Q) calculations consisted of six valence orbitals that correlate with the $1 \mathrm{~s}$ shells of the hydrogen atoms and the $2 s$ and $2 p$ shells of the oxygen atom. In analogy to active orbitals, the lowest-energy molecular orbital ( $\sim 1 \mathrm{~s}$ orbital of oxygen) was optimized in
CASSCF calculations, but unlike active orbitals that change occupancies it remained doubly occupied in all reference determinants defining the CASSCF and MRCI(Q) wave functions. Since our ab initio results are compared with the spectroscopically accurate ES surface of Ref. [102] and since it is well known that core electrons can significantly affect energy differences between different points on the potential energy surface, including the water potential [109, 117, 125], all electrons were correlated in the CC, CR-CC, and CASSCF-based $\operatorname{MRCI}(\mathrm{Q})$ calculations. This distinguishes our calculations from Refs. [109,125], in which the effects of core electrons were added as the a posteriori corrections to the results of valence-electron calculations.

The calculations were performed with the aug-cc-pCV XZ basis sets with $X=\mathrm{D}, \mathrm{T}, \mathrm{Q}$ [98-101], in which additional tight functions are added to the valence basis sets of the aug-cc-pVXZ quality to improve the description of core and core-valence correlation effects. The CCSD, CCSD(T), CCSD(TQ),b, CR-CCSD(T), CR-CCSD(TQ),a, CRCCSD(TQ),b, CR-CC(2,3),A, CR-CC(2,3), CR-CC(2,3)+ $\mathrm{Q}(\mathrm{a})$, and $\mathrm{CR}-\mathrm{CC}(2,3)+\mathrm{Q}(\mathrm{b})$ calculations were performed with the system of CC/CR-CC computer codes described in Refs. [20,30,41,89] and incorporated in the GAMESS package [90]. The MRCI $(\mathrm{Q})$ calculations were performed with the MOLPRO package [126]. In addition to the series of $\operatorname{MRCI}(\mathrm{Q})$ calculations using the aug-cc-pCVXZ basis sets with $X=\mathrm{D}, \mathrm{T}, \mathrm{Q}$, we performed the high accuracy $\operatorname{MRCI}(\mathrm{Q})$ calculations using the aug-cc-pCV5Z basis, to determine if the aug-cc-pCVQZ results are reasonably well converged. We could not perform such calculations using the $\mathrm{CC}$ and $\mathrm{CR}-\mathrm{CC}$ methods, since the atomic integral package used by GAMESS is restricted to $g$ functions and the aug-cc-pCV5Z basis contains $h$ functions which we did not want to drop in an ad hoc manner. Fortunately, we do not observe substantial changes in the results, when going from the aug-cc-pCVQZ to aug-cc-pCV5Z basis sets, which could alter our main conclusions. In order to facilitate our presentation, we refer to the aug-cc-pCVXZ basis set by mentioning the value of it's cardinal number $X(X=2$ for aug-cc-pCVDZ, $X=3$ for aug-cc-pCVTZ, $X=4$ for augcc-pCVQZ, and $X=5$ for aug-cc-pCV5Z). For example, we write "the $X=3$ basis set" instead of "the aug-cc-pCVTZ basis set." Instead of writing "the CR-CC $(2,3)$ calculations with the aug-cc-pCVTZ basis set," we simply write "the CR-CC $(2,3) / X=3$ calculations."

\section{Numerical results and discussion: probing potential energy surface of the water molecule with the conventional and renormalized coupled-cluster methods}

The results of our calculations are summarized in Tables 2 to 9. Table 2 serves as a reference for the remaining tables, 
Table 2 The ES and MRCI(Q) energies for the three potential energy surface cuts of water examined in this study: (i) the dissociation of a single $\mathrm{O}-\mathrm{H}$ bond, (ii) the $C_{2 v}$-symmetric dissociation of both $\mathrm{O}-\mathrm{H}$ bonds, and (iii) the $C_{2 v}$ dissociation into $\mathrm{H}_{2}\left(X^{1} \Sigma_{g}^{+}\right)$and $\mathrm{O}\left(2 p^{4}{ }^{1} D\right)$

\begin{tabular}{|c|c|c|c|c|c|c|}
\hline \multirow[t]{2}{*}{$R$} & \multirow[t]{2}{*}{$\alpha$} & \multirow[t]{2}{*}{ ES } & \multicolumn{4}{|c|}{$\operatorname{MRCI}(\mathrm{Q})$} \\
\hline & & & $X=2$ & $X=3$ & $X=4$ & $X=5$ \\
\hline \multicolumn{7}{|c|}{$\mathrm{H}_{2} \mathrm{O}\left(\tilde{X}^{1} A_{1}\right) \rightarrow \mathrm{H}\left(1 s^{2} S\right)+\mathrm{OH}\left(X^{2} \Pi\right)$} \\
\hline $1.25 R_{e}$ & 104.501 & 7367 & 6753 & 7177 & 7354 & 7396 \\
\hline $1.50 R_{e}$ & 104.501 & 18862 & 17945 & 18733 & 18990 & 19059 \\
\hline $1.75 R_{e}$ & 104.501 & 28857 & 27478 & 28510 & 28838 & 28928 \\
\hline $2.00 R_{e}$ & 104.501 & 35583 & 34067 & 35226 & 35615 & 35721 \\
\hline $2.50 R_{e}$ & 104.501 & 41024 & 40030 & 41277 & 41736 & 41857 \\
\hline $3.00 R_{e}$ & 104.501 & 42816 & 41521 & 42811 & 43282 & 43407 \\
\hline $4.00 R_{e}$ & 104.501 & 43779 & 41938 & 43250 & 43723 & 43848 \\
\hline $5.00 R_{e}$ & 104.501 & 43887 & 41970 & 43281 & 43754 & 43879 \\
\hline \multicolumn{7}{|c|}{$\mathrm{H}_{2} \mathrm{O}\left(\tilde{X}^{1} A_{1}\right) \rightarrow 2 \mathrm{H}\left(1 s^{2} S\right)+\mathrm{O}\left(2 p^{4}{ }^{3} P\right)$} \\
\hline $1.25 R_{e}$ & 104.501 & 14366 & 13119 & 14027 & 14380 & 14464 \\
\hline $1.50 R_{e}$ & 104.501 & 36152 & 34506 & 36188 & 36706 & 36843 \\
\hline $1.75 R_{e}$ & 104.501 & 54629 & 52359 & 54555 & 55224 & 55407 \\
\hline $2.00 R_{e}$ & 104.501 & 67354 & 64408 & 66912 & 67730 & 67950 \\
\hline $2.50 R_{e}$ & 104.501 & 78170 & 74341 & 77126 & 78113 & 78374 \\
\hline $3.00 R_{e}$ & 104.501 & 80585 & 76298 & 79196 & 80207 & 80478 \\
\hline $4.00 R_{e}$ & 104.501 & 81191 & 76792 & 79732 & 80752 & 81020 \\
\hline $5.00 R_{e}$ & 104.501 & 81219 & 76840 & 79776 & 80795 & 81062 \\
\hline \multirow[t]{2}{*}{$Y$} & $\alpha$ & ES & \multicolumn{4}{|c|}{ MRCI(Q) } \\
\hline & & & $X=2$ & $X=3$ & $X=4$ & $X=5$ \\
\hline \multicolumn{7}{|c|}{$\mathrm{H}_{2} \mathrm{O}\left(\tilde{X}^{1} A_{1}\right) \rightarrow \mathrm{H}_{2}\left(X^{1} \Sigma_{g}^{+}\right)+\mathrm{O}\left(2 p^{4}{ }^{1} D\right)$} \\
\hline 0.80 & 78.808 & 4998 & 4501 & 4809 & 4981 & 5023 \\
\hline 0.90 & 69.118 & 10660 & 9888 & 10374 & 10639 & 10701 \\
\hline 1.00 & 61.113 & 17927 & 16642 & 17326 & 17666 & 17745 \\
\hline 1.10 & 56.072 & 26589 & 23953 & 24887 & 25287 & 25383 \\
\hline 1.20 & 53.429 & 36326 & 31497 & 32699 & 33148 & 33260 \\
\hline 1.30 & 48.314 & 43726 & 38301 & 39623 & 40095 & 40215 \\
\hline 1.50 & 38.432 & 52137 & 48794 & 50171 & 50662 & 50786 \\
\hline 1.75 & 29.171 & 57311 & 55342 & 56604 & 57116 & 57236 \\
\hline 2.00 & 22.855 & 58486 & 56770 & 57663 & 58181 & 58294 \\
\hline 4.00 & 10.589 & 58696 & 57534 & 58058 & 58590 & 58694 \\
\hline
\end{tabular}

$R$ is an $\mathrm{O}-\mathrm{H}$ distance defining the dissociating $\mathrm{O}-\mathrm{H}$ bond(s), $Y$ (in $\AA$ ) is the distance between $\mathrm{O}$ and the line connecting both $\mathrm{H}$ nuclei, and $\alpha$ (in degree) is the $\mathrm{H}-\mathrm{O}-\mathrm{H}$ angle. The equilibrium values of $R, Y$, and $\alpha$ are $R_{e}=0.95785 \AA, Y_{e}=0.58641 \AA$, and $\alpha_{e}=104.501$ degree [109]. All energies $E$ (in $\left.\mathrm{cm}^{-1}\right)$ are reported as $E-E\left(R_{e}, \alpha_{e}\right)$, where $E\left(R_{e}, \alpha_{e}\right)$ are the corresponding values of $E$ at the equilibrium geometry. $X$ is a cardinal number defining the aug-cc-pCVXZ basis sets used in the MRCI(Q) calculations. In all MRCI(Q) calculations, all electrons were correlated

providing the MRCI(Q) and ES energies along the three dissociation pathways considered in our calculations. As one can see, the three potential energy surface cuts probe different energy regions. The single $\mathrm{O}-\mathrm{H}$ bond dissociation is characterized by the lowest energies (always reported in this work relative to the corresponding energies at the equilibrium geometry of Ref. [109], so that all energies are 0 at the equilibrium geometry), which do not exceed $44,000 \mathrm{~cm}^{-1}$. The $C_{2 v}$-symmetric dissociation pathway that leads to the
$\mathrm{H}_{2}\left(\begin{array}{ll}X & { }^{1} \Sigma_{g}^{+}\end{array}\right)$and $\mathrm{O}\left(2 p^{4}{ }^{1} D\right)$ products goes to higher energies, on the order of $59,000 \mathrm{~cm}^{-1}$, and the highest energies, on the order of $81,000 \mathrm{~cm}^{-1}$, are reached, when the simultaneous dissociation of both $\mathrm{O}-\mathrm{H}$ bonds is examined. The results in Table 2 show that the large scale MRCI(Q) calculations with the $X=5$ basis set agree with the ES potential, to within $\sim 10-200 \mathrm{~cm}^{-1}$, in the $R_{e} \leq R \leq 2 R_{e}$ and $R \geq 4 R_{e}$ regions of cut (i), $R_{e} \leq R<1.5 R_{e}$ and $R \geq 2.5 R_{e}$ regions of cut (ii), and $Y_{e} \leq Y \leq 1.0 \AA$ and $Y \geq 1.75 \AA$ 
Table 3 The dissociation of a single $\mathrm{O}-\mathrm{H}$ bond in water (into $\mathrm{H}\left(1 s^{2} S\right)+\mathrm{OH}\left(X^{2} \Pi\right)$; cut(i))

\begin{tabular}{|c|c|c|c|c|c|c|c|c|c|c|c|c|c|c|c|}
\hline \multirow[t]{2}{*}{$R$} & \multicolumn{3}{|l|}{ CCSD } & \multicolumn{3}{|c|}{$\operatorname{CCSD}(\mathrm{T})$} & \multicolumn{3}{|c|}{ CR-CCSD $(T)$} & \multicolumn{3}{|c|}{ CR-CC(2,3), $\mathrm{A}^{\mathrm{a}}$} & \multicolumn{3}{|c|}{ CR-CC $(2,3)$} \\
\hline & $=2$ & $=3$ & $=4$ & $=2$ & $X=3$ & $=4$ & $=2$ & $X=3$ & $X=4$ & $=2$ & $X=3$ & $X=4$ & $X=2$ & $X=3$ & $X=$ \\
\hline $25 R_{e}$ & 33 & 3 & 7662 & 6 & 7181 & 7251 & 815 & 17 & 7410 & 25 & 0 & 7389 & 810 & 216 & 735 \\
\hline $50 R_{e}$ & & & 19691 & & & & & & & & & & & & 08 \\
\hline $75 R_{e}$ & 3610 & 7 & 0 & 7 & 29 & 28844 & 27843 & 2 & 2 & 27764 & & & & 6 & 902 \\
\hline $00 R_{e}$ & 35889 & 37303 & 37739 & 4109 & 5231 & 66 & 3 & 3 & & & 0 & 36 & 5 & 7 & 596 \\
\hline $50 R_{e}$ & 43451 & 45308 & 45904 & 363 & 40628 & 41117 & 4 & 42687 & 43205 & & 6 & 42 & 9 & 39 & 4246 \\
\hline $00 R_{e}$ & 000 & 158 & 836 & 387 & 40600 & 7 & 42828 & 37 & 93 & & 96 & 95 & 47 & 17 & 4409 \\
\hline $00 R_{e}$ & 47145 & 49527 & 50262 & 3 & 38518 & 38938 & 43188 & 6 & 4 & & & & & & 44 \\
\hline $00 R_{e}$ & 47325 & 49760 & 505 & & & & 43107 & 2 & & & & & & & 437 \\
\hline & \multicolumn{3}{|c|}{ CCSD(TQ), b } & \multicolumn{3}{|c|}{ CR-CCSD(TQ),a } & \multicolumn{3}{|c|}{ CR-CCSD(TQ),b } & \multicolumn{3}{|c|}{$\mathrm{CR}-\mathrm{CC}(2,3)+\mathrm{Q}(\mathrm{a})^{b}$} & \multicolumn{3}{|c|}{$\mathrm{CR}-\mathrm{CC}(2,3)+\mathrm{Q}(\mathrm{b})^{b}$} \\
\hline & 2 & 3 & $X=4$ & 2 & 3 & 4 & & 3 & 4 & & & 4 & 2 & $=3$ & $X=$ \\
\hline 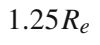 & & & & & 197 & & & & 7372 & & & 4 & & 8 & 73 \\
\hline $50 R_{e}$ & 17965 & 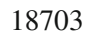 & . & 6 & ה & . & & 1 & & & & & & & 1897 \\
\hline $75 R_{e}$ & 27461 & 28446 & 28764 & 2700 & 8689 & 29012 & 2764 & 2868 & 2901 & 27466 & & 843 & & 9 & 2884 \\
\hline $00 R_{e}$ & 33948 & 35098 & 35491 & 34420 & 35659 & 36060 & 34398 & 35643 & 36047 & 34 & & & 37 & & 35701 \\
\hline $50 R_{e}$ & 39145 & 40432 & 40 & & 42340 & & 4 & 42297 & & 10 & 2 & & 14 & 49 & 20 \\
\hline $00 R_{e}$ & 39234 & 40445 & 40928 & 42482 & 44162 & 5 & 42432 & 19 & 76 & 1 & 11 & 16 & 151 & 43098 & 4367 \\
\hline $00 R_{e}$ & 37630 & 38536 & 38941 & 42842 & 580 & 45140 & 42845 & 44591 & 54 & 59 & 38 & 44042 & 41763 & 43449 & 405 \\
\hline $5.00 R_{e}$ & 36964 & 37713 & 38075 & 42735 & 44460 & 45016 & 42798 & 44536 & 45095 & 41690 & 43339 & 43941 & 41754 & 43416 & 4402 \\
\hline
\end{tabular}

One of the two $\mathrm{O}-\mathrm{H}$ bonds and the $\mathrm{H}-\mathrm{O}-\mathrm{H}$ angle are kept fixed at their respective equilibrium values taken from $\mathrm{Ref}$. [109] $\left(R_{e}=0.95785\right.$ $\AA$ and $\alpha_{e}=104.501$ degree, respectively). $R$ is an O-H distance defining the dissociating O-H bond. All energies $E$ (in $\mathrm{cm}^{-1}$ ) are reported as $E-E\left(R_{e}, \alpha_{e}\right)$, where $E\left(R_{e}, \alpha_{e}\right)$ are the corresponding values of $E$ at the equilibrium geometry. $X$ is a cardinal number defining the aug-cc-pCV $X Z$ basis sets used in the calculations. In all $\mathrm{CC}$ calculations, all electrons were correlated

${ }^{\text {a }}$ Equivalent to the $\operatorname{CCSD}(2)_{T}$ approach of Ref. [38]

${ }^{\mathrm{b}} \mathrm{CR}-\mathrm{CC}(2,3)+\mathrm{Q}(x)=\mathrm{CR}-\mathrm{CC}(2,3)+[\mathrm{CR}-\mathrm{CCSD}(\mathrm{TQ}), x-\mathrm{CR}-\mathrm{CCSD}(\mathrm{T})], x=\mathrm{a}, \mathrm{b}$

of cut (iii) ( $R$ is the $\mathrm{O}-\mathrm{H}$ separation for the dissociating $\mathrm{O}-\mathrm{H}$ bond or bonds; the meaning of $Y$ has been explained in Sect. 2). The $\mathrm{MRCI}(\mathrm{Q}) / X=4$ results are not much different, producing the results that in most cases do not differ from the $\operatorname{MRCI}(\mathrm{Q}) / X=5$ results by more than $100-200 \mathrm{~cm}^{-1}$ in the higher energy regions. Thus, with an exception of the region of intermediate $R$ and $Y$ values $\left(2 R_{e}<\right.$ $R<4 R_{e}$ for cut (i), $1.5 R_{e} \leq R<2.5 R_{e}$ for cut (ii), and $1.0 \AA<Y<1.75 \AA$ for cut (iii), the $\mathrm{MRCI}(\mathrm{Q}) / X=4$ or $\mathrm{MRCI}(\mathrm{Q}) / X=5$ and $\mathrm{ES}$ potential functions agree very well. Clearly, they cannot perfectly agree, since MRCI(Q) is not the exact theory. Moreover, our MRCI(Q) calculations are the standard non-relativistic calculations, ignoring relativistic, non-adiabatic, and quantum electrodynamical effects which all contribute to the water potential energy surface [109]. Besides, the minimum energy path defining cut (iii) determined from the ES function is certainly not identical to the similar path that would result from the MRCI(Q) calculations and there may be other small differences in the details of the $\operatorname{MRCI}(\mathrm{Q}) / X=4$ or $\operatorname{MRCI}(\mathrm{Q}) / X=5$ and ES potentials. On the other hand, the differences between the $\mathrm{MRCI}(\mathrm{Q}) / X=4$ or $\mathrm{MRCI}(\mathrm{Q}) / X=5$ and $\mathrm{ES}$ potential energy surfaces in the aforementioned regions of intermediate $R$ and $Y$ values, and intermediate or higher, but not the highest energies, which are as much as $700-800 \mathrm{~cm}^{-1}$ for cut (i) in the $R \approx 2.5 R_{e}$ region, $600-800 \mathrm{~cm}^{-1}$ for cut (ii) in the $R \approx 1.75 R_{e}$ region, and 3,500-3,600 $\mathrm{cm}^{-1}$ for cut (iii) in the $Y \approx 1.3 \AA$ A region, cannot be explained by the mere neglect of the relativistic, non-adiabatic, and quantum electrodynamical effects. They indicate that either the MRCI(Q) approach is insufficiently accurate or the ES potential needs further refinement. The former is always possible, but the latter explanation would not be a complete surprise either, since we must remember that the ES potential function is constructed by combining the many-body expansion potential of Murrell and Carter [115] and the polynomial potential form of Polyansky, Jensen, and Tennyson [116]. This makes the ES potential very accurate around the minimum, up to about $19,000 \mathrm{~cm}^{-1}$, and in the asymptotic regions, including the $\mathrm{H}\left(1 s^{2} S\right)+\mathrm{OH}\left(X^{2} \Pi\right)$, $2 \mathrm{H}\left(1 s^{2} S\right)+\mathrm{O}\left(2 p^{4}{ }^{3} P\right)$, and $\mathrm{H}_{2}\left(X^{1} \Sigma_{g}^{+}\right)+\mathrm{O}\left(2 p^{4}{ }^{1} D\right)$ asymptotes examined in this study, but the accuracy of the ES potential function in the regions of intermediate internuclear separations and energies which connect the spectroscopic and asymptotic regions of the water potential energy 
Table 4 The $C_{2 v}$-symmetric double dissociation of water (into $2 \mathrm{H}\left(1 s^{2} S\right)+\mathrm{O}\left(2 p^{4}{ }^{3} P\right)$; cut (ii))

\begin{tabular}{|c|c|c|c|c|c|c|c|c|c|c|c|c|c|c|c|}
\hline \multirow[t]{2}{*}{$R$} & \multicolumn{3}{|l|}{ CCSD } & \multicolumn{3}{|c|}{$\operatorname{CCSD}(\mathrm{T})$} & \multicolumn{3}{|c|}{ CR-CCSD(T) } & \multicolumn{3}{|c|}{ CR-CC(2,3), $\mathrm{A}^{\mathrm{a}}$} & \multicolumn{3}{|c|}{ CR-CC $(2,3)$} \\
\hline & $=2$ & $=3$ & -4 & $=2$ & $K=3$ & $X=4$ & $=2$ & $X=3$ & $X=4$ & $=2$ & $X=3$ & $X=4$ & $X=2$ & $X=3$ & $X=$ \\
\hline 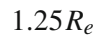 & & 14684 & (04) & 212 & 14041 & 14381 & & 14191 & 4 & 10 & 77 & 56 & 226 & 100 & 445 \\
\hline $50 R_{e}$ & & & & & & & & & & & & & & & 36960 \\
\hline $75 R_{e}$ & 55003 & 57497 & & & 54646 & & & 55626 & & & & & & & 577 \\
\hline $00 R_{e}$ & 68471 & 716 & 7 & 3 & 6666 & 6 & 0 & & & & 67973 & & 9 & & 343 \\
\hline $50 R_{e}$ & 79497 & 84194 & 573 & 039 & 70640 & 71855 & 36 & 79223 & 42 & 329 & 75866 & 2 & 027 & 96 & 7614 \\
\hline $00 R_{e}$ & 79696 & 8 & 6853 & 39 & 59917 & 74 & 36 & 79026 & 64 & 9964 & 73450 & 60 & 38 & 92 & 7361 \\
\hline $00 R_{e}$ & 8228 & 8 & 85708 & 3548 & 45309 & 4 & 9 & 3 & 7 & 9 & 70653 & & & & 11 \\
\hline $00 R_{e}$ & 77699 & 8 & & & 40815 & & 71808 & 6 & & & 7 & & & & 23 \\
\hline & \multicolumn{3}{|c|}{ CCSD(TQ),b } & \multicolumn{3}{|c|}{ CR-CCSD(TQ), a } & \multicolumn{3}{|c|}{ CR-CCSD(TQ),b } & \multicolumn{3}{|c|}{$\mathrm{CR}-\mathrm{CC}(2,3)+\mathrm{Q}(\mathrm{a})^{b}$} & \multicolumn{3}{|c|}{$\mathrm{CR}-\mathrm{CC}(2,3)+\mathrm{Q}(\mathrm{b})^{b}$} \\
\hline & & & & & 3 & $X$ & & & 4 & & $=3$ & 4 & & 3 & - \\
\hline & & & & & & & & & & & & & & & 14 \\
\hline $50 R_{e}$ & 34552 & 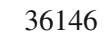 & - & 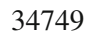 & s. & & & 36403 & & & & & & & 36 \\
\hline $75 R_{e}$ & 52314 & ונדוד & 000 & 20 & 14 & & 52892 & 55142 & & & & & & 8 & 5529 \\
\hline $00 R_{e}$ & 64074 & 66549 & 67364 & $65-101$ & 68219 & & 65560 & & & & 66 & & & & $0 / 04$. \\
\hline $50 R_{e}$ & 75356 & 76491 & 198 & 337 & 80006 & & 86 & 3 & & 77 & 9 & 33 & 26 & 26 & 7772 \\
\hline $00 R_{e}$ & 93104 & 88747 & 88276 & 038 & 83153 & 01 & 900 & 70 & 77 & 73140 & 18 & & 77003 & 79635 & 8073 \\
\hline $00 R_{e}$ & 126095 & 115610 & 773 & 41 & 86600 & 11 & 48 & 89 & 07 & 37 & 25 & 33 & 79544 & 115 & 3529 \\
\hline $.00 R_{e}$ & 139384 & 127204 & 124945 & 84989 & 89436 & 90773 & 86028 & 90109 & 91361 & 78923 & 82253 & 83260 & 79962 & 82926 & 8384 \\
\hline
\end{tabular}

The $\mathrm{H}-\mathrm{O}-\mathrm{H}$ angle is kept fixed at its equilibrium value taken from Ref. [109] ( $\alpha_{e}=104.501$ degree). $R$ is an $\mathrm{O}-\mathrm{H}$ distance and $R_{e}=0.95785$ $\AA$ is the equilibrium value of $R$ [109]. All energies $E\left(\mathrm{in} \mathrm{cm}^{-1}\right)$ are reported as $E-E\left(R_{e}, \alpha_{e}\right)$, where $E\left(R_{e}, \alpha_{e}\right)$ are the corresponding values of $E$ at the equilibrium geometry. $X$ is a cardinal number defining the aug-cc-pCVXZ basis sets used in the calculations. In all CC calculations, all electrons were correlated

${ }^{\text {a }}$ Equivalent to the $\operatorname{CCSD}(2)_{T}$ approach of Ref. [38]

${ }^{\mathrm{b}} \mathrm{CR}-\mathrm{CC}(2,3)+\mathrm{Q}(x)=\mathrm{CR}-\mathrm{CC}(2,3)+[\mathrm{CR}-\mathrm{CCSD}(\mathrm{TQ}), x-\mathrm{CR}-\mathrm{CCSD}(\mathrm{T})], x=\mathrm{a}, \mathrm{b}$

surface is less certain. A comparison of the $\mathrm{MRCI}(\mathrm{Q})$ and best CR-CC data may help us to decide if the ES potential needs further improvements in the regions of intermediate $R$ or $Y$ values and energies that connect the region of the global minimum with the asymptotes.

The main CC and CR-CC results for the three potential energy surface cuts examined in this work (the CC and CR-CC energies calculated relative to the corresponding energies at the minimum taken from Ref. [109]) are given in Tables 3, 4, 5. In order to facilitate our analysis, we give in Tables 6, 7, 8 the differences between the CC/CR-CC and the corresponding $\mathrm{MRCI}(\mathrm{Q})$ energies for each of the three augcc-pCV XZ basis sets used in the CC/CR-CC calculations. In Table 9, we compare the errors in the best CR-CC $(2,3)+Q(b) /$ $X=4$ results, relative to the $\operatorname{MRCI}(\mathrm{Q}) / X=4$ data, with the differences between the CR-CC $(2,3)+\mathrm{Q}(\mathrm{b}) / X=4$ and ES energies and the analogous differences between the $\operatorname{MRCI}(\mathrm{Q}) / X=4,5$ and $\mathrm{ES}$ energies.

A close inspection of Tables 3 to 8, particularly Tables 6 to 8 , allows us to appreciate the nature of the challenges the single-reference CC methods face when describing global potential energy surfaces along bond breaking coordinates.
It also allows us to reemphasize the usefulness of the three dissociation pathways that we chose in this study to test the $\mathrm{CC}$ and CR-CC methods, which create different types of bond breaking or bond stretching situations. Indeed, the relatively large differences between the CCSD and $\mathrm{MRCI}(\mathrm{Q})$ energies, which exceed $\sim 300-700 \mathrm{~cm}^{-1}$ for small stretches of the O-H bond(s) $\left(R \approx 1.25 R_{e}-1.5 R_{e}\right.$ in the case of cut (i), $R \approx 1.25 R_{e}$ in the case of cut (ii), and $Y \approx 0.8-0.9 \AA$ in the case of cut (iii)), and huge differences between the CCSD and MRCI(Q) energies, on the order of 4,000-7,000 $\mathrm{cm}^{-1}$, in the $R \geq 3 R_{e}$ region of cut (i) and $R \approx 2 R_{e}-3 R_{e}$ region of cut (ii), and more than $1,000 \mathrm{~cm}^{-1}$ for larger $Y$ values in the case of cut (iii), clearly show that one needs to include higher-than-doubly excited clusters in the CC calculations to obtain reasonable results. Not surprisingly, the CCSD approach is qualitatively correct in the case of cut (i), which corresponds to single-bond breaking (which is, in the zero-order approximation, a two-electron process), producing errors relative to $\mathrm{MRCI}(\mathrm{Q})$ which monotonically increase with $R$, while being completely erratic in the case of the double $\mathrm{O}-\mathrm{H}$ dissociation defining cut (ii), producing a large hump in the region of intermediate $R$ values. The CCSD 
Table 5 The $C_{2 v}$ dissociation of water into $\mathrm{H}_{2}\left(X^{1} \Sigma_{g}^{+}\right)$and $\mathrm{O}\left(2 p^{4}{ }^{1} D\right)$ (cut (iii))

\begin{tabular}{|c|c|c|c|c|c|c|c|c|c|c|c|c|c|c|c|c|}
\hline \multirow[t]{2}{*}{ Y } & \multirow[t]{2}{*}{$\alpha$} & \multicolumn{3}{|l|}{ CCSD } & \multicolumn{3}{|c|}{$\operatorname{CCSD}(\mathrm{T})$} & \multicolumn{3}{|c|}{ CR-CCSD(T) } & \multicolumn{3}{|c|}{$\mathrm{CR}-\mathrm{CC}(2,3), \mathrm{A}^{\mathrm{a}}$} & \multicolumn{3}{|c|}{ CR-CC $(2,3)$} \\
\hline & & $X=2$ & $X=3$ & $X=4$ & $X=2$ & $X=3$ & $X=4$ & $X=2$ & $X=3$ & $X=4$ & $X=2$ & $X=3$ & $X=4$ & $X=2$ & $X=3$ & $X=$ \\
\hline 80 & 78.808 & 4774 & 5126 & 5303 & 4555 & 4847 & 5017 & 4597 & 4904 & 5074 & 4579 & 4880 & 5049 & 4558 & 4867 & 5044 \\
\hline .90 & 69.118 & 10376 & 10928 & 11201 & 9977 & 10432 & 10691 & 10056 & 10536 & 10797 & 10022 & 10492 & 10752 & 992 & 10468 & 0744 \\
\hline 1.00 & 61.113 & 17384 & 18147 & 18499 & 5770 & 17407 & 17739 & 97 & 17568 & 17 & 44 & 17500 & 3 & 89 & 17470 & 7827 \\
\hline 1.10 & 56.072 & 24974 & 25995 & 26411 & 24122 & 24997 & 25385 & 24310 & 25228 & 25621 & 24240 & 25135 & 25525 & 4155 & 25088 & 5514 \\
\hline 1.20 & 53.429 & 32808 & 34103 & 34568 & 31700 & 32836 & 33268 & 31969 & 33158 & 33597 & 31887 & 33039 & 33471 & 31771 & 32963 & 33438 \\
\hline 1.30 & 48.314 & 39726 & 41129 & 41615 & 38513 & 39783 & 40238 & 38830 & 40155 & 40618 & 38760 & 40031 & 40483 & 38617 & 39923 & 40416 \\
\hline 1.50 & 38.432 & 49953 & 51355 & 51853 & 48998 & 50382 & 50864 & 49259 & 50675 & 51163 & 49231 & 50586 & 51060 & 49051 & 50433 & 50943 \\
\hline .75 & 29.171 & 55899 & 57130 & 57642 & 55485 & 56838 & 57358 & 55596 & 56941 & 57462 & 55595 & 56915 & 57430 & 55444 & 56787 & 57331 \\
\hline .00 & 22.855 & 57688 & 58693 & 59243 & 35 & 58525 & 59102 & 57444 & 58609 & 59180 & 57456 & 58615 & 59 & 57299 & 58503 & 59083 \\
\hline .00 & 10.589 & 59495 & 60316 & 60926 & 38 & 59825 & 3 & 06 & 60031 & 60672 & 59037 & 60063 & $60^{\circ}$ & 58768 & 59872 & 60543 \\
\hline \multirow[t]{2}{*}{ Y } & & \multicolumn{3}{|c|}{ CCSD(TQ),b } & \multicolumn{3}{|c|}{ CR-CCSD(TQ), a } & \multicolumn{3}{|c|}{ CR-CCSD(TQ),b } & \multicolumn{3}{|c|}{$\mathrm{CR}-\mathrm{CC}(2,3)+\mathrm{Q}(\mathrm{a})^{\mathrm{b}}$} & \multicolumn{3}{|c|}{$\mathrm{CR}-\mathrm{CC}(2,3)+\mathrm{Q}(\mathrm{b})^{\mathrm{b}}$} \\
\hline & & $X=2$ & $X=3$ & $X=4$ & $=2$ & $=3$ & $X=4$ & $X$ & 3 & $X=4$ & 2 & $x$ & $x-4$ & 2 & $=3$ & $t=$ \\
\hline 80 & 78.808 & 4535 & 4838 & 5008 & 4549 & 4858 & 5029 & 4551 & 4861 & 5032 & 4510 & 4821 & 4999 & 4513 & 4824 & 5002 \\
\hline 90 & 69.118 & 9945 & 10416 & 10676 & 9969 & 10452 & 10715 & 9973 & 10458 & 10721 & 9905 & 10385 & 10662 & 9909 & 10390 & 10668 \\
\hline .00 & 61.113 & 16727 & 17385 & 17717 & 16767 & 17443 & 17779 & 16772 & 17450 & 17 & 59 & 17 & 03 & 64 & 17352 & 17711 \\
\hline 10 & 56.072 & 2 & 2 & 2 & 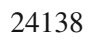 & 2 & 7 & 0 & 2 & 4 & 3 & 1 & & 6 & 26 & 56 \\
\hline .20 & 53.429 & 31631 & 32797 & 33234 & 31765 & 32958 & 33400 & 9 & 3 & 3 & 8 & 4 & 2 & 2 & 63 & 42 \\
\hline .30 & 48.314 & 38454 & 39757 & 40 & 3 & 35 & 40 & 33 & 64 & 41 & 42 & 17 & 42 & 20 & 39732 & 40229 \\
\hline 1.50 & 38.432 & 48996 & 50402 & 50891 & 49211 & 50615 & 01 & 77 & 584 & 51072 & 49003 & 50372 & 50881 & 48970 & 50341 & 50851 \\
\hline 1.75 & 29.171 & 55500 & 56837 & 57361 & 55636 & 56968 & 57487 & 55605 & 56937 & 57457 & 55484 & 56814 & 57356 & 55452 & 56783 & 57326 \\
\hline 2.00 & 22.855 & 57312 & 58468 & 59047 & 57498 & 58653 & 59225 & 57463 & 58618 & 59191 & 57354 & 58548 & 59128 & 57318 & 58512 & 59094 \\
\hline 4.00 & 10.589 & 58689 & 59724 & 60384 & 59036 & 60058 & 60701 & 59014 & 60025 & 60670 & 58798 & 59899 & 60572 & 58776 & 59866 & 60541 \\
\hline
\end{tabular}

The approximate minimum energy path, determined using the potential function of Ref. [102], is defined by the coordinate $Y$ (in $\AA$ ), which is the distance between $\mathrm{O}$ and the line connecting both $\mathrm{H}$ nuclei, and the $\mathrm{H}-\mathrm{O}-\mathrm{H}$ angle $\alpha$ (in degree). The equilibrium values of $Y$ and $\alpha$ are $Y_{e}=0.58641$ $\AA$ and $\alpha_{e}=104.501$ degree [109]. All energies $E\left(\right.$ in cm$\left.^{-1}\right)$ are reported as $E-E\left(Y_{e}, \alpha_{e}\right)$, where $E\left(Y_{e}, \alpha_{e}\right)$ are the corresponding values of $E$ at the equilibrium geometry. $X$ is a cardinal number defining the aug-cc-pCVXZ basis sets used in the calculations. In all CC calculations, all electrons were correlated

${ }^{\text {a }}$ Equivalent to the $\operatorname{CCSD}(2)_{T}$ approach of Ref. [38]

${ }^{\mathrm{b}} \mathrm{CR}-\mathrm{CC}(2,3)+\mathrm{Q}(x)=\mathrm{CR}-\mathrm{CC}(2,3)+[\mathrm{CR}-\mathrm{CCSD}(\mathrm{TQ}), x-\mathrm{CR}-\mathrm{CCSD}(\mathrm{T})], x=\mathrm{a}, \mathrm{b}$

approach is also erratic in the case of cut (iii), in which two $\mathrm{O}-\mathrm{H}$ bonds have to be significantly stretched during the formation of the $\mathrm{H}_{2}\left(X^{1}{ }^{1} \Sigma_{g}^{+}\right)$and $\mathrm{O}\left(2 p^{4}{ }^{1} D\right)$ products, although the errors relative to $\mathrm{MRCI}(\mathrm{Q})$ are not as large in this case as in the other two cuts. One of the reasons is that unlike cuts (i) and (ii), which lead to the fragmentations of the closed-shell water molecule on the singlet ground-state surface into open-shell (doublet or even triplet) products, which introduce very large non-dynamic correlation effects, the minimum energy path that defines cut (iii) leads to the formation of the closed-shell $\left(\mathrm{H}_{2}\left(X^{1} \Sigma_{g}^{+}\right)\right)$or singlet $\left(\mathrm{O}\left(2 p^{4}{ }^{1} D\right)\right)$ fragments while the $\mathrm{O}-\mathrm{H}$ bonds are broken. Moreover, the hydrogen product molecule is a two-electron system, which is described exactly by the CCSD approach. These differences between cuts (i) and (ii), on the one hand, and cut (iii), on the other hand, can be seen by examining the largest doubly excited $\left(T_{2}\right)$ cluster amplitudes. The largest spin-free $T_{2}$ amplitude, which corresponds to the HOMO $\rightarrow$ LUMO double excitation at $R=5 R_{e}$ of cut (i) equals, according to the $\mathrm{CCSD} / X=4$ calculations, -0.858278 (this is a singlebond breaking case, so other $T_{2}$ amplitudes are much smaller). The two largest $T_{2}$ amplitudes, which correspond to the $\mathrm{HOMO} \rightarrow$ LUMO and $(\mathrm{HOMO}-1) \rightarrow(\mathrm{LUMO}+1)$ double excitations at $R=5 R_{e}$ of cut (ii) equal, according to the $\mathrm{CCSD} / X=4$ calculations, -0.774880 and -0.774382 , respectively (clearly, there are a few other large $T_{2}$ amplitudes in this case, which engage the highest two occupied and lowest two unoccupied orbitals, since this is a double dissociation of water into $2 \mathrm{H}\left(1 s^{2} S\right)+\mathrm{O}\left(2 p^{4}{ }^{3} P\right)$ that involves, in the zero-order description, four active orbitals and four electrons). For comparison, the largest $T_{2}$ amplitudes along the minimum energy path defining cut (iii) never exceed 
Table 6 The differences between CC/CR-CC energies, calculated relative to their equilibrium values (the CC/CR-CC $\left[E-E\left(R_{e}, \alpha_{e}\right)\right]$ values in Table 3 ) and the corresponding $\operatorname{MRCI}(\mathrm{Q})$ relative energies (the
$\operatorname{MRCI}(\mathrm{Q})\left[E-E\left(R_{e}, \alpha_{e}\right)\right]$ values in Table 2) for the dissociation of a single $\mathrm{O}-\mathrm{H}$ bond in water (into $\mathrm{H}\left(1 s^{2} S\right)+\mathrm{OH}\left(X^{2} \Pi\right)$; cut (i))

\begin{tabular}{|c|c|c|c|c|c|c|c|c|c|c|c|c|c|c|c|}
\hline \multirow[t]{2}{*}{$R$} & \multicolumn{3}{|l|}{ CCSD } & \multicolumn{3}{|c|}{$\operatorname{CCSD}(\mathrm{T})$} & \multicolumn{3}{|c|}{ CR-CCSD(T) } & \multicolumn{3}{|c|}{ CR-CC(2,3), $\mathrm{A}^{\mathrm{a}}$} & \multicolumn{3}{|c|}{ CR-CC $(2,3)$} \\
\hline & $X=2$ & $X=3$ & $X=4$ & $X=2$ & $X=3$ & $X=4$ & $=2$ & $X=3$ & $X=4$ & $X=2$ & $X=3$ & $X=4$ & $X=2$ & $X=3$ & $X=4$ \\
\hline $.25 R_{e}$ & 280 & 306 & 308 & 43 & 4 & -3 & 92 & 70 & 65 & 72 & 42 & 35 & 57 & 39 & 39 \\
\hline $1.50 R_{e}$ & 642 & 695 & 701 & 78 & 11 & -2 & 206 & 176 & 167 & 160 & 110 & 97 & 118 & 96 & 97 \\
\hline $1.75 R_{e}$ & 1132 & 1253 & 1270 & 89 & 19 & 6 & 365 & 363 & 356 & 286 & 243 & 227 & 175 & 186 & 186 \\
\hline $2.00 R_{e}$ & 1822 & 2077 & 2124 & 42 & 5 & 5 & 608 & 687 & 698 & 487 & 490 & 483 & 248 & 321 & 351 \\
\hline $2.50 R_{e}$ & 3421 & 4031 & 4168 & -667 & -649 & -619 & 1115 & 1410 & 1469 & 889 & 1019 & 1039 & 369 & 662 & 725 \\
\hline $3.00 R_{e}$ & 4479 & 5347 & 5554 & -2134 & -2211 & -2195 & 1307 & 1726 & 1811 & 997 & 1185 & 1213 & 326 & 706 & 813 \\
\hline $4.00 R_{e}$ & 5207 & 6277 & 6539 & -4385 & -4732 & -4785 & 1250 & 1726 & 1818 & 871 & 1068 & 1092 & 168 & 584 & 720 \\
\hline $5.00 R_{e}$ & 5355 & 6479 & 6754 & -5211 & -5689 & -5779 & 1137 & 1611 & 1699 & 746 & 934 & 95 & 93 & 490 & 625 \\
\hline & \multicolumn{3}{|c|}{ CCSD(TQ),b } & \multicolumn{3}{|c|}{ CR-CCSD(TQ),a } & \multicolumn{3}{|c|}{ CR-CCSD(TQ),b } & \multicolumn{3}{|c|}{$\mathrm{CR}-\mathrm{CC}(2,3)+\mathrm{Q}(\mathrm{a})^{\mathrm{b}}$} & \multicolumn{3}{|c|}{$\mathrm{CR}-\mathrm{CC}(2,3)+\mathrm{Q}(\mathrm{b})^{\mathrm{b}}$} \\
\hline & $X=2$ & $X=3$ & $X=4$ & $X=2$ & $X=3$ & $X=4$ & $X=2$ & $X=3$ & $X=4$ & $X=2$ & $X=3$ & $X=4$ & $X=2$ & $X=3$ & $X=4$ \\
\hline $1.25 R_{e}$ & 18 & -10 & -16 & 40 & 20 & 16 & 4 & 22 & 18 & 5 & -11 & -10 & 6 & -9 & -8 \\
\hline $1.50 R_{e}$ & 20 & -30 & -41 & 91 & 64 & 56 & 90 & 65 & 58 & 2 & -16 & -14 & 1 & -15 & -12 \\
\hline $1.75 R_{e}$ & -17 & -64 & -74 & 178 & 179 & 174 & 170 & 175 & 172 & -12 & 2 & 5 & -21 & -1 & 2 \\
\hline $2.00 R_{e}$ & -119 & -128 & -124 & 353 & 433 & 445 & 331 & 417 & 432 & -7 & 67 & 99 & -30 & 51 & 86 \\
\hline $2.50 R_{e}$ & -885 & -845 & -811 & 781 & 1063 & 1122 & 729 & 1020 & 1084 & 36 & 315 & 378 & -16 & 272 & 339 \\
\hline $3.00 R_{e}$ & -2287 & -2366 & -2354 & 961 & 1351 & 1433 & 911 & 1308 & 1394 & -20 & 330 & 434 & -70 & 287 & 395 \\
\hline $4.00 R_{e}$ & -4308 & -4714 & -4782 & 904 & 1330 & 1417 & 907 & 1341 & 1431 & -179 & 188 & 319 & -175 & 199 & 333 \\
\hline $5.00 R_{e}$ & -5006 & -5568 & -5679 & 765 & 1179 & 1262 & 828 & 1255 & 1341 & -280 & 58 & 187 & -216 & 135 & 267 \\
\hline
\end{tabular}

$X$ is a cardinal number defining the aug-cc-pCVXZ basis sets used in the calculations

${ }^{a}$ Equivalent to the $\operatorname{CCSD}(2)_{T}$ approach of Ref. [38]

${ }^{\mathrm{b}} \mathrm{CR}-\mathrm{CC}(2,3)+\mathrm{Q}(x)=\mathrm{CR}-\mathrm{CC}(2,3)+[\mathrm{CR}-\mathrm{CCSD}(\mathrm{TQ}), x-\mathrm{CR}-\mathrm{CCSD}(\mathrm{T})], x=\mathrm{a}, \mathrm{b}$

$\approx 0.2$ and are usually considerably smaller. This explains the observed differences in the performance of the CCSD and various CCSD-based non-iterative $\mathrm{CC}$ methods in the calculations for cut (iii), where the overall behavior of the single-reference CC methods, although not perfect, remains quite reasonable, and the other two cuts examined in this work, where the standard CC approximations, including $\operatorname{CCSD}, \operatorname{CCSD}(\mathrm{T})$, and $\operatorname{CCSD}(\mathrm{TQ}), \mathrm{b}$, display dramatic failures.

The above discussion confirms the known fact that one has to go beyond the basic CCSD approximation and account for higher-than-doubly excited clusters to obtain a quantitatively accurate description of the potential energy surface, even in the vicinity of the equilibrium geometry. This is often done with the $\operatorname{CCSD}(\mathrm{T})$ approach, which describes the leading effects due to triply excited clusters via non-iterative corrections to the CCSD energy. As shown in our tables, the $\operatorname{CCSD}(\mathrm{T})$ approach provides excellent results that almost perfectly agree with the results of $\mathrm{MRCI}(\mathrm{Q})$ calculations, when stretches of the $\mathrm{O}-\mathrm{H}$ bonds are small. For example, in the case of cut (i), the differences between the $\operatorname{CCSD}(\mathrm{T}) / X=$ 4 and $\operatorname{MRCI}(\mathrm{Q}) / X=4$ energies do not exceed $6 \mathrm{~cm}^{-1}$ up to
$R=2 R_{e}$ and for cut (ii) they remain smaller than $38 \mathrm{~cm}^{-1}$ up to $R=1.5 R_{e}$. This is impressive, if we realize that the $R \approx 2 R_{e}$ and $R \approx 1.5 R_{e}$ regions of cuts (i) and (ii), respectively, are characterized by the energies of $\approx 36,000 \mathrm{~cm}^{-1}$. A similarly impressive performance of $\operatorname{CCSD}(\mathrm{T})$ is observed for other aug-cc-pCV XZ basis sets, although we should note a rather substantial error increase, relative to $\mathrm{MRCI}(\mathrm{Q})$, when the $X=2$ basis sets is employed (particularly for cuts (i) and (ii); cf. Tables 6 and 7). Interestingly enough, in the case of cut (ii), the $\operatorname{CCSD}(\mathrm{T})$ results remain reasonable up to $\sim 67,000$ $\mathrm{cm}^{-1}$ or $R \approx 2 R_{e}$ (unsigned errors relative to $\operatorname{MRCI}(\mathrm{Q}$ ) on the order of $200-300 \mathrm{~cm}^{-1}$ ). One has to be very careful, however, in interpreting these high accuracies obtained with $\operatorname{CCSD}(\mathrm{T})$ for small and, in the case of cut (ii), intermediate stretches of the $\mathrm{O}-\mathrm{H}$ bonds, particularly that $\mathrm{CCSD}(\mathrm{T})$ eventually suffers significant breakdowns and it may not always be easy to predict when one should stop trusting the $\operatorname{CCSD}(\mathrm{T})$ approach. Indeed, if we correct the $\operatorname{CCSD}(\mathrm{T})$ results for the dominant effects due to quadruply excited clusters, as is done by the CCSD(TQ),b approach, which can only improve the quality of $\mathrm{CC}$ calculations in non-degenerate regions of the potential energy surface, the agreement between the $\mathrm{CC}$ 
Table 7 The differences between CC/CR-CC energies, calculated relative to their equilibrium values (the CC/CR-CC $\left[E-E\left(R_{e}, \alpha_{e}\right)\right]$ values in Table 4) and the corresponding $\operatorname{MRCI}(\mathrm{Q})$ relative energies (the
$\operatorname{MRCI}(\mathrm{Q})\left[E-E\left(R_{e}, \alpha_{e}\right)\right]$ values in Table 2) for the $C_{2 v}$-symmetric double dissociation of water (into $2 \mathrm{H}\left(1 s^{2} S\right)+\mathrm{O}\left(2 p^{4}{ }^{3} P\right)$; cut (ii))

\begin{tabular}{|c|c|c|c|c|c|c|c|c|c|c|c|c|c|c|c|}
\hline \multirow[t]{2}{*}{$R$} & \multicolumn{3}{|l|}{ CCSD } & \multicolumn{3}{|c|}{$\operatorname{CCSD}(\mathrm{T})$} & \multicolumn{3}{|c|}{ CR-CCSD(T) } & \multicolumn{3}{|c|}{$\mathrm{CR}-\mathrm{CC}(2,3), \mathrm{A}^{a}$} & \multicolumn{3}{|c|}{$\mathrm{CR}-\mathrm{CC}(2,3)$} \\
\hline & $X=2$ & $=3$ & $=4$ & $X=2$ & $X=3$ & $X=4$ & $X=2$ & $X=3$ & $X=4$ & $X=2$ & $X=3$ & $X=4$ & $X=2$ & $X=3$ & $X=4$ \\
\hline $25 R_{e}$ & 600 & 657 & 662 & 93 & 14 & 1 & 206 & 164 & 154 & 160 & 100 & 86 & 107 & 73 & 79 \\
\hline $50 R_{e}$ & 1466 & 1594 & 1609 & 189 & 65 & 3 & 0 & 488 & 469 & 2 & 313 & 286 & 274 & 233 & 254 \\
\hline $75 R_{e}$ & 2644 & 2942 & 2984 & 197 & 91 & 66 & 1028 & 1071 & 1059 & 741 & 690 & 660 & 474 & 538 & 547 \\
\hline $00 R_{e}$ & 4063 & 4703 & 4808 & -285 & -246 & -232 & 1612 & 1889 & 1917 & 949 & 1061 & 1059 & 381 & 647 & 709 \\
\hline $50 R_{e}$ & 5156 & 7068 & 7460 & -7302 & -6486 & -6258 & 845 & 2097 & 2329 & -2012 & -1260 & -1111 & -3314 & -2230 & -1971 \\
\hline $00 R_{e}$ & 3398 & 6065 & 6646 & -19659 & -19279 & -19133 & -1862 & -170 & 157 & -6334 & -5746 & -5647 & -7760 & -6904 & -6589 \\
\hline $00 R_{e}$ & 1436 & 4321 & 4956 & -33244 & -34423 & -34697 & -4293 & -2579 & -2258 & -9433 & -9079 & -9052 & -10497 & -9854 & -9636 \\
\hline $00 R_{e}$ & 859 & 3750 & 4388 & -37103 & -38961 & 12 & -5032 & 60 & 19 & -10291 & -9999 & 85 & -11098 & -10542 & -10563 \\
\hline \multirow[t]{2}{*}{$R$} & \multicolumn{3}{|c|}{$\operatorname{CCSD}(\mathrm{TQ}), \mathrm{b}$} & \multicolumn{3}{|c|}{ CR-CCSD(TQ),a } & \multicolumn{3}{|c|}{ CR-CCSD(TQ),b } & \multicolumn{3}{|c|}{$\mathrm{CR}-\mathrm{CC}(2,3)+\mathrm{Q}(\mathrm{a})^{b}$} & \multicolumn{3}{|c|}{$\mathrm{CR}-\mathrm{CC}(2,3)+\mathrm{Q}(\mathrm{b})^{b}$} \\
\hline & $X=2$ & $K=3$ & $X=4$ & & & & $X=2$ & & & $X=2$ & & $X=4$ & & $X=3$ & $X=4$ \\
\hline $25 R_{e}$ & 8 & -17 & -29 & 8 & 5 & 44 & 93 & 58 & 50 & -10 & -39 & -31 & -6 & -34 & -25 \\
\hline $.50 R_{e}$ & 46 & -42 & -65 & 243 & 207 & 191 & 246 & 215 & 202 & -13 & -49 & -24 & -10 & -40 & -13 \\
\hline $.75 R_{e}$ & -45 & -118 & -138 & 548 & 589 & 580 & 533 & 587 & 582 & -7 & 56 & 69 & -21 & 53 & 71 \\
\hline $2.00 R_{e}$ & -334 & -363 & -366 & 1073 & 1307 & 1329 & 1061 & 1296 & 1323 & -158 & 64 & 121 & -170 & 53 & 115 \\
\hline $2.50 R_{e}$ & 1015 & -635 & -915 & 1996 & 2880 & 3040 & 3345 & 3827 & 3908 & -2164 & -1447 & -1260 & -815 & -500 & -391 \\
\hline $3.00 R_{e}$ & 16806 & 9551 & 8069 & 2740 & 3957 & 4194 & 6602 & 7174 & 7270 & -3158 & -2778 & -2553 & 705 & 439 & 523 \\
\hline $4.00 R_{e}$ & 49303 & 35878 & 33021 & 5449 & 6868 & 7159 & 8956 & 9957 & 10155 & -755 & -407 & -219 & 2752 & 2683 & 2777 \\
\hline $5.00 R_{e}$ & 62544 & 47428 & 44150 & 8149 & 9660 & 9978 & 9188 & 10333 & 10566 & 2083 & 2477 & 2465 & 3122 & 3150 & 3053 \\
\hline
\end{tabular}

$X$ is a cardinal number defining the aug-cc-pCVXZ basis sets used in the calculations

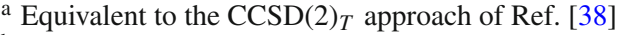

${ }^{\mathrm{b}} \mathrm{CR}-\mathrm{CC}(2,3)+\mathrm{Q}(x)=\mathrm{CR}-\mathrm{CC}(2,3)+[\mathrm{CR}-\mathrm{CCSD}(\mathrm{TQ}), x-\mathrm{CR}-\mathrm{CCSD}(\mathrm{T})], x=\mathrm{a}, \mathrm{b}$

and $\mathrm{MRCI}(\mathrm{Q})$ results in the $R \leq 2 R_{e}$ regions of cuts (i) and (ii) is no longer as impressive as in the $\operatorname{CCSD}(\mathrm{T})$ case. For example, the -2 and $5 \mathrm{~cm}^{-1}$ differences between the $\operatorname{CCSD}(\mathrm{T}) / X=4$ and $\mathrm{MRCI}(\mathrm{Q}) / X=4$ energies obtained for cut (i) at $R=1.5 R_{e}$ and $2 R_{e}$ increase to -41 and $-124 \mathrm{~cm}^{-1}$, respectively, when the CCSD(TQ),b method is employed. The 1 and $38 \mathrm{~cm}^{-1}$ differences between the $\operatorname{CCSD}(\mathrm{T}) / X=4$ and $\operatorname{MRCI}(\mathrm{Q}) / X=4$ energies obtained for cut (ii) at $R=1.25 R_{e}$ and $1.5 R_{e}$ increase, in absolute value, to 29 and $65 \mathrm{~cm}^{-1}$, when instead of $\operatorname{CCSD}(\mathrm{T})$ we use the CCSD(TQ),b method. This means that either the $\operatorname{CCSD}(\mathrm{T})$ results are very accurate due to fortuitous cancellation of errors or the MRCI(Q) results that we use as a benchmark are not as accurate as the CCSD(TQ),b results in the region of smaller stretches of the $\mathrm{O}-\mathrm{H}$ bonds, where CCSD(TQ), b can be safely applied, creating a false impression about superb accuracy of the CCSD(T) approximation. Clearly, both interpretations are possible. For example, the $\operatorname{CCSD}(\mathrm{T})$ approach is known to provide the results which are often better than those obtained with the full CCSDT method, which makes no physical sense whatsoever. We believe that in the case of water the CCSD(TQ),b approach is more accurate than $\mathrm{MRCI}(\mathrm{Q})$ in the spectroscopic region and moderate stretches of the $\mathrm{O}-\mathrm{H}$ bond(s), although both methods provide high quality results. Our belief is based on the fact that the CCSD(TQ),b method includes higher-order dynamical correlation effects, which dominate in the spectroscopic region and which are not present in $\mathrm{MRCI}(\mathrm{Q})$. The overall superiority of $\operatorname{CCSD}(\mathrm{TQ})$, b over MRCI(Q) for moderate stretches of the $\mathrm{O}-\mathrm{H}$ bond(s) can be seen by comparing the differences between the $\operatorname{CCSD}(\mathrm{TQ}), \mathrm{b} / X=4$ and $\mathrm{ES}$ energies in the $R \leq 2 R_{e}$ regions of cuts (i) and (ii) with the corresponding differences between the MRCI $(\mathrm{Q}) / X=4$ and ES energies. This illustrates the well-known advantage of using the CC methods over MRCI techniques, which are not as effective in accounting for the dynamical correlation effects that dominate electron correlations near the equilibrium geometry as the high-level CC approaches. Interestingly enough, further increase of the basis set makes the agreement between the MRCI $(\mathrm{Q})$ and ES surfaces in the region of smaller stretches of the $\mathrm{O}-\mathrm{H}$ bond(s), where the ES potential function is nearly spectroscopic, even worse (cf. the $\mathrm{MRCI}(\mathrm{Q}) / X=$ 4 and $\mathrm{MRCI}(\mathrm{Q}) / X=5$ results in Table 9). The superiority of the $\operatorname{CCSD}(\mathrm{TQ}), \mathrm{b}$ and related $\operatorname{CCSD}\left(\mathrm{TQ}_{\mathrm{f}}\right)$ methods, 
Table 8 The differences between CC/CR-CC energies, calculated relative to their equilibrium values (the CC/CR-CC $\left[E-E\left(R_{e}, \alpha_{e}\right)\right]$ values in Table 5) and the corresponding $\operatorname{MRCI}(\mathrm{Q})$ relative energies (the $\operatorname{MRCI}(\mathrm{Q})\left[E-E\left(R_{e}, \alpha_{e}\right)\right]$ values in Table 2$)$ for the $C_{2 v}$ dissociation of water into $\mathrm{H}_{2}\left(X^{1} \Sigma_{g}^{+}\right)$and $\mathrm{O}\left(2 p^{4}{ }^{1} D\right)$ along the approximate minimum energy path determined using the potential function of Ref. [102] (cut (iii))

\begin{tabular}{|c|c|c|c|c|c|c|c|c|c|c|c|c|c|c|c|c|}
\hline \multirow[t]{2}{*}{$Y$} & \multirow[t]{2}{*}{$\alpha$} & \multicolumn{3}{|l|}{ CCSD } & \multicolumn{3}{|c|}{$\operatorname{CCSD}(\mathrm{T})$} & \multicolumn{3}{|c|}{ CR-CCSD(T) } & \multicolumn{3}{|c|}{ CR-CC(2,3), $\mathrm{A}^{\mathrm{a}}$} & \multicolumn{3}{|c|}{ CR-CC $(2,3)$} \\
\hline & & $X=2$ & $X=3$ & $X=4$ & $X=2$ & $X=3$ & $X=4$ & $X=2$ & $X=3$ & $X=4$ & $X=2$ & $X=3$ & $X=4$ & $X=2$ & $X=3$ & $X=4$ \\
\hline 80 & 78.808 & 273 & 317 & 322 & 54 & 38 & 36 & 96 & 95 & 93 & 78 & 71 & 68 & 57 & 58 & 63 \\
\hline .90 & 69.118 & 488 & 554 & 562 & 89 & 58 & 52 & 168 & 162 & 158 & 134 & 118 & 113 & 104 & 94 & 105 \\
\hline 1.00 & 61.113 & 742 & 821 & 833 & 128 & 81 & 73 & 255 & 242 & 237 & 202 & 174 & 167 & 147 & 144 & 161 \\
\hline 1.10 & 56.072 & 1021 & 1108 & 1124 & 169 & 110 & 98 & 357 & 341 & 334 & 287 & 248 & 238 & 202 & 201 & 227 \\
\hline 1.20 & 53.429 & 1311 & 1404 & 1420 & 203 & 137 & 120 & 472 & 459 & 449 & 390 & 340 & 323 & 274 & 264 & 290 \\
\hline 1.30 & 48.314 & 1425 & 1506 & 1520 & 212 & 160 & 143 & 529 & 532 & 523 & 459 & 408 & 388 & 316 & 300 & 321 \\
\hline 1.50 & 38.432 & 1159 & 1184 & 1191 & 204 & 211 & 202 & 465 & 504 & 501 & 437 & 415 & 398 & 257 & 262 & 281 \\
\hline 1.75 & 29.171 & 557 & 526 & 526 & 143 & 234 & 242 & 254 & 337 & 346 & 253 & 311 & 314 & 102 & 183 & 215 \\
\hline .00 & 22.855 & 918 & 1030 & 1062 & 565 & 862 & 921 & 674 & 946 & 999 & 686 & 952 & 1005 & 529 & 840 & 902 \\
\hline .00 & 10.589 & 1961 & 2258 & 2336 & 1204 & 1767 & 1893 & 1472 & 1973 & 2082 & 1503 & 2005 & 2114 & 1234 & 1814 & 1953 \\
\hline & \multirow[t]{2}{*}{$\alpha$} & \multicolumn{3}{|c|}{ CCSD(TQ),b } & \multicolumn{3}{|c|}{ CR-CCSD(TQ),a } & \multicolumn{3}{|c|}{ CR-CCSD(TQ),b } & \multicolumn{3}{|c|}{$\mathrm{CR}-\mathrm{CC}(2,3)+\mathrm{Q}(\mathrm{a})^{b}$} & \multicolumn{3}{|c|}{$\mathrm{CR}-\mathrm{CC}(2,3)+\mathrm{Q}(\mathrm{b})^{b}$} \\
\hline & & $X=2$ & $X=3$ & $X=4$ & $X=2$ & $X=3$ & $X=4$ & $X=2$ & $X=3$ & $X=4$ & $X=2$ & $X=3$ & $X=4$ & $X=2$ & $X=3$ & $X=4$ \\
\hline 80 & 78.808 & 34 & 29 & 27 & 48 & 49 & 48 & 50 & 52 & 51 & 9 & 12 & 18 & 12 & 15 & 21 \\
\hline .90 & 69.118 & 57 & 42 & 37 & 81 & 78 & 76 & 85 & 84 & 82 & 17 & 11 & 23 & 21 & 16 & 29 \\
\hline 1.00 & 61.113 & 85 & 59 & 51 & 125 & 117 & 113 & 130 & 124 & 121 & 17 & 19 & 37 & 22 & 26 & 45 \\
\hline 1.10 & 56.072 & 114 & 79 & 70 & 185 & 174 & 170 & 187 & 180 & 177 & 30 & 34 & 62 & 33 & 39 & 69 \\
\hline 1.20 & 53.429 & 134 & 98 & 86 & 268 & 259 & 252 & 262 & 258 & 253 & 71 & 65 & 94 & 65 & 64 & 94 \\
\hline 1.30 & 48.314 & 153 & 134 & 123 & 354 & 356 & 349 & 332 & 341 & 335 & 141 & 124 & 147 & 119 & 109 & 134 \\
\hline 1.50 & 38.432 & 202 & 231 & 229 & 417 & 444 & 439 & 383 & 413 & 410 & 209 & 201 & 219 & 176 & 170 & 189 \\
\hline 1.75 & 29.171 & 158 & 233 & 245 & 294 & 364 & 371 & 263 & 333 & 341 & 142 & 210 & 240 & 110 & 179 & 210 \\
\hline 2.00 & 22.855 & 542 & 805 & 866 & 728 & 990 & 1044 & 693 & 955 & 1010 & 584 & 885 & 947 & 548 & 849 & 913 \\
\hline 4.00 & 10.589 & 1155 & 1666 & 1794 & 1502 & 2000 & 2111 & 1480 & 1967 & 2080 & 1264 & 1841 & 1982 & 1242 & 1808 & 1951 \\
\hline
\end{tabular}

$X$ is a cardinal number defining the aug-cc-pCVXZ basis sets used in the calculations

${ }^{a}$ Equivalent to the $\operatorname{CCSD}(2)_{T}$ approach of Ref. [38]

${ }^{\mathrm{b}} \mathrm{CR}-\mathrm{CC}(2,3)+\mathrm{Q}(x)=\mathrm{CR}-\mathrm{CC}(2,3)+[\mathrm{CR}-\mathrm{CCSD}(\mathrm{TQ}), x-\mathrm{CR}-\mathrm{CCSD}(\mathrm{T})], x=\mathrm{a}, \mathrm{b}$

which account for triply and quadruply excited clusters, over $\operatorname{CCSD}(\mathrm{T})$, which ignores the latter clusters, in applications involving potential energy surfaces near the equilibrium geometry is well-documented as well (cf., e.g., Refs. [11-13, $19,20,30,32,57,127-129]$ ) and our calculations confirm this superiority, in spite of the tiny (and misleading) differences between the $\operatorname{CCSD}(\mathrm{T})$ and $\operatorname{MRCI}(\mathrm{Q})$ energies for cuts (i) and (ii).

Before discussing the failures of the $\operatorname{CCSD}(\mathrm{T})$ and CCSD(TQ),b methods at larger $\mathrm{O}-\mathrm{H}$ separations of cuts (i) and (ii) and improvements offered by the CR-CC methods, which are quite apparent when we examine the results shown in Tables 3 to 8 , let us point out that in the case of cut (iii), the behavior of the $\operatorname{CCSD}(\mathrm{T})$ and $\operatorname{CCSD}(\mathrm{TQ}), \mathrm{b}$ methods vs. the $\operatorname{MRCI}(\mathrm{Q})$ approach is somewhat different, when compared to the other two cuts explored in this work. As already pointed out above, the doubly excited (also, singly excited) cluster amplitudes along the minimum energy path defining cut (iii) never become large and the corresponding $\mathrm{H}_{2}\left(X^{1} \Sigma_{g}^{+}\right)$and $\mathrm{O}\left(2 p^{4} D\right)$ dissociation products are of the closed-shell $\left(\mathrm{H}_{2}\left(X^{1} \Sigma_{g}^{+}\right)\right)$or open-shell singlet $\left(\mathrm{O}\left(2 p^{4}{ }^{1} D\right)\right)$ type. Thus, it is not completely surprising to observe the relatively good performance of the standard single-reference $\operatorname{CCSD}(\mathrm{T})$ and $\operatorname{CCSD}(\mathrm{TQ}), \mathrm{b}$ approaches in the entire region of $Y$ values shown in our tables (with the exception, perhaps, of the last two points, $Y=2.0$ and $4.0 \AA$, although we must remember that these points are located at more than $58,000 \mathrm{~cm}^{-1}$ above the global minimum, which makes the $\sim 500-1,900 \mathrm{~cm}^{-1}$ errors relatively small, particularly for the relatively inexpensive single-reference calculations using the RHF reference; cf. the discussion below for the additional remarks). In particular, the differences between the $\operatorname{CCSD}(\mathrm{T})$ and $\mathrm{MRCI}(\mathrm{Q})$ energies do not exceed $250 \mathrm{~cm}^{-1}$ in the entire $Y_{e} \leq Y \leq$ $1.75 \AA$ region, in which energies become as large as 
Table 9 The differences between the CR-CC(2,3)+Q(b) and MRCI(Q) energies obtained with the aug-cc-pCVQZ $(X=4)$ basis, the differences between the CR-CC $(2,3)+Q(b) / a u g-c c-p C V Q Z$ and $E S$ energies, and the differences between the MRCI $(\mathrm{Q}) /$ aug-cc-pCVXZ $(X=4,5)$ and ES energies (all energies calculated relative to their corresponding equilibrium values, as in the earlier tables) for the three cuts of the water potential energy surface: (i) the dissociation of a single O-H bond, (ii) the $C_{2 v}$-symmetric dissociation of both $\mathrm{O}-\mathrm{H}$ bonds, and (iii) the $C_{2 v}$ dissociation into $\mathrm{H}_{2}\left(X^{1} \Sigma_{g}^{+}\right)$and $\mathrm{O}\left(2 p^{4}{ }^{1} D\right)$ (see Table 2 for the definitions of $R$ and $Y$, the corresponding values of the $\mathrm{H}-\mathrm{O}-\mathrm{H}$ angle $\alpha$, and the equilibrium values of $R, Y$, and $\alpha$ ). All energy differences are in $\mathrm{cm}^{-1}$

\begin{tabular}{|c|c|c|c|c|}
\hline$R$ & $\begin{array}{l}\mathrm{CR}-\mathrm{CC}(2,3)+\mathrm{Q}(\mathrm{b}) / X=4 \\
-\mathrm{MRCI}(\mathrm{Q}) / X=4\end{array}$ & $\begin{array}{l}\mathrm{CR}-\mathrm{CC}(2,3)+\mathrm{Q}(\mathrm{b}) / X=4 \\
-\mathrm{ES}\end{array}$ & $\begin{array}{l}\operatorname{MRCI}(\mathrm{Q}) / X=4 \\
-\mathrm{ES}\end{array}$ & $\begin{array}{l}\operatorname{MRCI}(\mathrm{Q}) / X=5 \\
-\mathrm{ES}\end{array}$ \\
\hline \multicolumn{5}{|c|}{$\mathrm{H}_{2} \mathrm{O}\left(\tilde{X}^{1} A_{1}\right) \rightarrow \mathrm{H}\left(1 s^{2} S\right)+\mathrm{OH}\left(X^{2} \Pi\right)$} \\
\hline $1.25 R_{e}$ & -8 & -21 & -13 & 29 \\
\hline $1.50 R_{e}$ & -12 & 116 & 128 & 197 \\
\hline $1.75 R_{e}$ & 2 & -17 & -19 & 71 \\
\hline $2.00 R_{e}$ & 86 & 118 & 32 & 138 \\
\hline $2.50 R_{e}$ & 339 & 1051 & 712 & 833 \\
\hline $3.00 R_{e}$ & 395 & 861 & 466 & 591 \\
\hline $4.00 R_{e}$ & 333 & 277 & -56 & 69 \\
\hline $5.00 R_{e}$ & 267 & 134 & -133 & -8 \\
\hline \multicolumn{5}{|c|}{$\mathrm{H}_{2} \mathrm{O}\left(\tilde{X}^{1} A_{1}\right) \rightarrow 2 \mathrm{H}\left(1 s^{2} S\right)+\mathrm{O}\left(2 p^{4}{ }^{3} P\right)$} \\
\hline $1.25 R_{e}$ & -25 & -11 & 14 & 98 \\
\hline $1.50 R_{e}$ & -13 & 541 & 554 & 691 \\
\hline $1.75 R_{e}$ & 71 & 666 & 595 & 778 \\
\hline $2.00 R_{e}$ & 115 & 491 & 376 & 596 \\
\hline $2.50 R_{e}$ & -391 & -448 & -57 & 204 \\
\hline $3.00 R_{e}$ & 523 & 145 & -378 & -107 \\
\hline $4.00 R_{e}$ & 2777 & 2338 & -439 & -171 \\
\hline $5.00 R_{e}$ & 3053 & 2629 & -424 & -157 \\
\hline$Y$ & $\begin{array}{l}\mathrm{CR}-\mathrm{CC}(2,3)+\mathrm{Q}(\mathrm{b}) / X=4 \\
-\mathrm{MRCI}(\mathrm{Q}) / X=4\end{array}$ & $\begin{array}{l}\mathrm{CR}-\mathrm{CC}(2,3)+\mathrm{Q}(\mathrm{b}) / X=4 \\
-\mathrm{ES}\end{array}$ & $\begin{array}{l}\operatorname{MRCI}(\mathrm{Q}) / X=4 \\
-\mathrm{ES}\end{array}$ & $\begin{array}{l}\operatorname{MRCI}(\mathrm{Q}) / X=5 \\
-\mathrm{ES}\end{array}$ \\
\hline \multicolumn{5}{|c|}{$\mathrm{H}_{2} \mathrm{O}\left(\tilde{X}^{1} A_{1}\right) \rightarrow \mathrm{H}_{2}\left(X^{1} \Sigma_{g}^{+}\right)+\mathrm{O}\left(2 p^{4}{ }^{1} D\right)$} \\
\hline 0.80 & 21 & 4 & -17 & 25 \\
\hline 0.90 & 29 & 8 & -21 & 41 \\
\hline 1.00 & 45 & -216 & -261 & -182 \\
\hline 1.10 & 69 & -1233 & -1302 & -1206 \\
\hline 1.20 & 94 & -3084 & -3178 & -3066 \\
\hline 1.30 & 134 & -3497 & -3631 & -3511 \\
\hline 1.50 & 189 & -1286 & -1475 & -1351 \\
\hline 1.75 & 210 & 15 & -195 & -75 \\
\hline 2.00 & 913 & 608 & -305 & -192 \\
\hline 4.00 & 1951 & 1845 & -106 & -2 \\
\hline
\end{tabular}

$\sim 57,000 \mathrm{~cm}^{-1}$ (recall that $Y_{e}=0.58641 \AA$ ), and are smaller than $100 \mathrm{~cm}^{-1}$ when $Y$ does not exceed $1.1 \AA$ (energies below $\sim 27,000 \mathrm{~cm}^{-1}$ ). The CCSD(TQ), b approach reduces these differences even further, showing a nice and systematic behavior of the single-reference CC theory in this case. Clearly, it is interesting to examine if the CR-CC approaches, which are primarily designed to improve the $\mathrm{CC}$ results when a system is fragmented into open-shell fragments, can maintain the high accuracies of the $\operatorname{CCSD}(\mathrm{T})$ and $\operatorname{CCSD}(\mathrm{TQ}), \mathrm{b}$ calculations and systematic improvements in the results when going from the triples to the quadruples levels of CC theory in the case of the minimum energy path that leads to the singlet, but non-closed-shell products.

One issue that we do not address in this study and that may affect the results of all CC and CR-CC calculations in the $Y \geq$ 2.0 ̊ region of cut (iii) (and, perhaps, the best CR-CC results in the $R>3 R_{e}$ region of cut (ii)) is the issue of the existence of the avoided crossings of ground and excited states 
of water at larger internuclear separations (see, e.g., Refs. $[113,114,118,119]$ and references therein). For example, it is possible that for the larger, but not the largest, values of $Y$ of cut (ii), where the $\mathrm{H}-\mathrm{H}$ bond is still somewhat stretched, other dissociation channels, such as $\mathrm{H}_{2}\left(b^{3} \Sigma_{u}^{+}\right)+\mathrm{O}\left(2 p^{4}{ }^{3} P\right)$, compete with the ground-state $\mathrm{H}_{2}\left(X^{1}{ }^{1} \Sigma_{g}^{+}\right)+\mathrm{O}\left(2 p^{4}{ }^{1} D\right)$ channel and that the CCSD solutions that we found, for example, at $Y=4.0 \AA$, are not necessarily the solutions that correlate with the lowest-energy state of water of the ${ }^{1} A_{1}$ symmetry. This would explain a steep increase in the errors of the CCSD(T), CCSD(TQ), b, and all, otherwise very accurate, CR-CC calculations in the $Y \geq 2.0 \AA$ region of cut (iii), from $\sim 200-400 \mathrm{~cm}^{-1}$ at $Y=1.75 \AA$ to $\sim 1,800-2,100$ $\mathrm{cm}^{-1}$ at $Y=4.0 \AA$. We tried to find other CCSD solutions in the $Y \geq 2.0 \AA$ region, but we have not been successful. Thus, it may be necessary to calculate other electronic states in the $Y \geq 2.0 \AA$ region, which one can try to do using, for example, the excited-states CR-CC methods, such as CR-EOMCCSD(T) [87,88], and the excited-state variant of CR-CC $(2,3)$ tested in Refs. [14,44], in which the properly renormalized triples corrections are added to the energies obtained in the equation-of-motion (EOM) CCSD calculations. It is not unusual to observe the switching between the ground and low-lying excited states of the same symmetry in CC/EOMCC calculations in the vicinity of avoided crossings [130]. The state-switching of this type would explain the larger errors in the $Y \geq 2.0 \AA$ region observed in our CC calculations. We will examine this issue in the future.

Much of the above discussion points to the importance of properly balancing various correlation effects and the need to account for the triple as well as quadruple excitations in the high quality calculations of molecular potential energy surfaces. The properly constructed theory should provide an accurate description of triply, quadruply, and, if need be, other higher-order clusters, without the fortuitous cancellation of errors that the $\operatorname{CCSD}(\mathrm{T})$ approach displays. Clearly, the good theory should also eliminate the significant failures of the $\operatorname{CCSD}(\mathrm{T}), \operatorname{CCSD}(\mathrm{TQ}), \mathrm{b}$, and other similar methods at larger internuclear separations or at least be more robust in this regard. In the case of the water potential examined in this work, these failures are dramatic. As shown in Tables 3 to 8 (particularly, Tables 6 to 8 ), the unsigned errors in the $\operatorname{CCSD}(\mathrm{T}) / X=2-4$ energies, relative to the corresponding $\operatorname{MRCI}(\mathrm{Q}) / X=2-4$ data, range between 619 and $5,779 \mathrm{~cm}^{-1}$, when the $R=2.5 R_{e}-5 R_{e}$ region of cut (i) is examined, and 6,258 and $39,412 \mathrm{~cm}^{-1}$, when the $R=$ $2.5 R_{e}-5 R_{e}$ region of cut (ii) is explored, and the CCSD(T) energies go considerably below the $\operatorname{MRCI}(\mathrm{Q})$ energies. The CCSD(TQ),b approach does not improve the situation at all, producing the $811-5,679 \mathrm{~cm}^{-1}$ unsigned errors, relative to $\operatorname{MRCI}(\mathrm{Q})$, in the $R=2.5 R_{e}-5 R_{e}$ region of cut (i) and the $635-62,544 \mathrm{~cm}^{-1}$ unsigned errors, relative to the corresponding MRCI(Q) data, in the $R=2.5 R_{e}-5 R_{e}$ region of cut (ii).
Unlike CCSD(T), the CCSD(TQ), b energies are significantly above the corresponding MRCI(Q) energies at larger $\mathrm{O}-\mathrm{H}$ separations of cut (ii), while being below the MRCI(Q) energies for cut (i). All of this demonstrates the divergent behavior of the standard single-reference $\mathrm{CC}$ methods, caused by the large non-dynamic correlation effects (which manifest themselves through large doubly excited cluster amplitudes, as described above), the poor description of the wave function by the CCSD approach, on which the $\operatorname{CCSD}(\mathrm{T})$ and CCSD(TQ),b methods rely, and the strongly divergent nature of the many-body perturbation theory (MBPT) series, on which the standard (T) and (TQ) energy corrections are based, in the regions of larger internuclear separations.

As shown in Tables 6 to 8, the CR-CCSD(T) method provides considerable improvements in the $\operatorname{CCSD}(\mathrm{T})$ results for the single-bond breaking defining cut (i), reducing, for example, the $4,385-4,785 \mathrm{~cm}^{-1}$ and $5,211-5,779 \mathrm{~cm}^{-1}$ errors in the $\operatorname{CCSD}(\mathrm{T}) / X=2-4$ energies at $R=4 R_{e}$ and $5 R_{e}$, relative to the corresponding $\operatorname{MRCI}(\mathrm{Q}) / X=2-4$ data, to $1,250-1,818 \mathrm{~cm}^{-1}$ and $1,137-1,699 \mathrm{~cm}^{-1}$, respectively. The CR-CCSD(TQ), a and CR-CCSD(TQ),b methods provide further improvements and a nice and smooth description of the entire cut (i), with an exception, perhaps, of the last point at $R=5 R_{e}$, where a small error reduction compared to $R=4 R_{e}$ may be a signature of the eventual problems somewhere in the $R \gg 5 R_{e}$ region, although we are not sure about it at this time. For example, the differences between the CR-CCSD(TQ),b $X=4$ and $\operatorname{MRCI}(\mathrm{Q}) / X=4$ energies smoothly increase with $R$, from 18 and $58 \mathrm{~cm}^{-1}$ at $R=1.25 R_{e}$ and $1.5 R_{e}$, respectively (energies on the order of 7,400 and $18,900 \mathrm{~cm}^{-1}$ ), to $432 \mathrm{~cm}^{-1}$ at $R=2 R_{e}$, and $1,431 \mathrm{~cm}^{-1}$ at $R=4 R_{e}$, where the energy is almost $44,000 \mathrm{~cm}^{-1}$. The situation for the more challenging cut (ii), where both $\mathrm{O}-\mathrm{H}$ bonds are broken, is, at least to some extent, similar to that observed in the case of cut (i), with the CRCCSD(TQ), a and CR-CCSD(TQ), b methods eliminating the pathological behavior of $\operatorname{CCSD}(\mathrm{T})$ and $\operatorname{CCSD}(\mathrm{TQ}), \mathrm{b}$ at larger stretches of both $\mathrm{O}-\mathrm{H}$ bonds. Again, we observe a smooth increase of the differences between the CR-CCSD(TQ), a or CR-CCSD(TQ),b and MRCI(Q) energies with $R$, from 44 (50) and $191(202) \mathrm{cm}^{-1}$ at $R=1.25 R_{e}$ and $1.5 R_{e}$, where energies are on the order of $\sim 14,000$ and $36,000 \mathrm{~cm}^{-1}$, respectively, through $1,329(1,323) \mathrm{cm}^{-1}$ at $R=2 R_{e}$, where the energy exceeds $67,000 \mathrm{~cm}^{-1}$, and $9,978(10,566) \mathrm{cm}^{-1}$ at $R=5 R_{e}$, where the energies exceed $81,000 \mathrm{~cm}^{-1}$, when the CR-CCSD(TQ), $\mathrm{a} / X=4$ (CR-CCSD(TQ), $\mathrm{b} / X=4$ ) results are examined. We do not claim that these are superb results, but it is quite encouraging to see that the CR-CC methodology is capable of providing significant improvements over the standard $\mathrm{CC}$ results, even when the double dissociation of water is examined. There is, of course, a difference between the behavior of the CR-CCSD(T) method in the case of the double $\mathrm{O}-\mathrm{H}$ dissociation defining cut (ii) and the 
single-bond breaking defining cut (i). The CR-CCSD(T) approach provides a reasonably smooth description of cut (i), while failing in the case of cut (ii). This is a consequence of ignoring the quadruply excited clusters in the CR$\operatorname{CCSD}(\mathrm{T})$ calculations, which are absolutely critical in cases of double bond breaking (while improving accuracies in the calculations for single-bond breaking). It is interesting to observe, though, the substantial improvements in the poor $\operatorname{CCSD(T)~results~in~the~} R=2.5 R_{e}-5 R_{e}$ region of cut (ii) by the CR-CCSD(T) approach (error reduction in the $X=4$ calculations at $R=5 R_{e}$, relative to the corresponding $\operatorname{MRCI}(\mathrm{Q}) / X=4$ result, from more than $39,000 \mathrm{~cm}^{-1}$ in the $\operatorname{CCSD}(\mathrm{T})$ case to $\approx 3,000 \mathrm{~cm}^{-1}$ in the $\operatorname{CR}-\operatorname{CCSD}(\mathrm{T})$ case). It is also interesting to observe that the CR-CCSD(T), CR-CCSD(TQ), a, and CR-CCSD(TQ), b approaches provide a relatively accurate and smooth description of the "easier" cut (iii) as well. In this case, as pointed out above, the conventional $\operatorname{CCSD}(\mathrm{T})$ and $\operatorname{CCSD}(\mathrm{TQ}), \mathrm{b}$ approximations work quite well, but it is good to see that the $\mathrm{CR}-\operatorname{CCSD}(\mathrm{T})$ and CR-CCSD(TQ), $x(x=\mathrm{a}, \mathrm{b})$ methods are, more or less, equally effective, with the CR-CCSD(TQ), $x$ approaches providing systematic improvements over the relatively good CRCCSD(T) results. The $\sim 90-530 \mathrm{~cm}^{-1}$ differences between the CR-CCSD(T) and MRCI $(\mathrm{Q})$ energies and the slightly smaller $\sim 50-440 \quad \mathrm{~cm}^{-1}$ differences between the CR-CCSD(TQ), $x$ and $\operatorname{MRCI}(\mathrm{Q})$ energies in the entire $Y=$ $0.8-1.75 \AA$ region, where energies grow from about 5,000 to more than $57,000 \mathrm{~cm}^{-1}$ above the global minimum, is clearly an encouraging result, confirming the usefulness of the CR-CC methods.

The CR-CCSD(T) and CR-CCSD(TQ), $x(x=\mathrm{a}, \mathrm{b})$ methods provide substantial improvements in the regions of larger internuclear separations, where the standard CCSD(T) and CCSD(TQ),b approaches fail, but it would be useful to achieve further error reduction in the CR-CC calculations, particularly in the cases of cuts (i) and (ii), which are more challenging for the single-reference $\mathrm{CC}$ methods than cut (iii). It would also be useful to improve the results of CR-CC calculations in the vicinity of the equilibrium region, where the $\operatorname{CCSD}(\mathrm{T})$ and $\operatorname{CCSD}(\mathrm{TQ}), \mathrm{b}$ methods are somewhat more accurate than the CR-CCSD(T) and CR-CCSD(TQ), $x(x=$ a, b) approaches. As explained in Refs. [41,42], the recently proposed $\mathrm{CR}-\mathrm{CC}(2,3)$ theory not only eliminates the small size extensivity errors from the CR-CCSD(T) results (which in the case of water are negligible, since water molecule is a small, 10-electron system), but it also improves the accuracy of CR-CCSD $(\mathrm{T})$ calculations by adding various product terms involving the singly and doubly excited clusters and the triply excited moments of the CCSD equations to the bare triexcited moment terms that are already present in the CR$\operatorname{CCSD}(\mathrm{T})$ triples correction formula [131]. It is, therefore, interesting to examine the performance of the $\mathrm{CR}-\mathrm{CC}(2,3)$ approach using the three cuts of the water potential examined in this work. Since we have already noticed the importance of quadruply excited clusters in improving the results, particularly for the double $\mathrm{O}-\mathrm{H}$ dissociation defining cut (ii), it is also useful to investigate if correcting the CR-CC(2,3) results for quadruples through the $\mathrm{CR}-\mathrm{CC}(2,3)+\mathrm{Q}(\mathrm{a})$ and $\mathrm{CR}-\mathrm{CC}(2,3)+\mathrm{Q}(\mathrm{b})$ methods described in the Introduction gives the desired high accuracies for a wide range of nuclear geometries of the water system explored in this work. Following the discussion of the CR-CC $(2,3)$ theory in Refs. $[41,42,44]$, we consider two variants of CR-CC $(2,3)$ in this study, namely, the complete variant $\mathrm{D}$, for which we continue to use an acronym $\mathrm{CR}-\mathrm{CC}(2,3)$ without additional letters, and the approximate variant $\mathrm{A}$, called $\mathrm{CR}-\mathrm{CC}(2,3), \mathrm{A}$, which is obtained by replacing the diagonal matrix elements of the similarity-transformed Hamiltonian of CCSD involving triply excited determinants, which enter the triples correction of CR-CC $(2,3)$, by the usual MBPT denominator defining triple excitations, i.e., $\left(\epsilon_{a}+\epsilon_{b}+\epsilon_{c}-\epsilon_{i}-\epsilon_{j}-\epsilon_{k}\right)$. The CR$\mathrm{CC}(2,3)$, A approach is equivalent to the $\operatorname{CCSD}(2)_{T}$ method of Ref. [38].

As shown in Tables 3 to 8 , the $\mathrm{CR}-\mathrm{CC}(2,3), \mathrm{A}$ $\left(=\operatorname{CCSD}(2)_{\mathrm{T}}\right)$ and full CR-CC $(2,3)(=\mathrm{CR}-\mathrm{CC}(2,3), \mathrm{D})$ approaches provide improvements in the $\operatorname{CR}-\operatorname{CCSD}(\mathrm{T})$ results for the single-bond breaking defining cut (i) and the $C_{2 v}$ dissociation pathway into $\mathrm{H}_{2}\left(X^{1} \Sigma_{g}^{+}\right)$and $\mathrm{O}\left(2 p^{4}{ }^{1} D\right)$ in the entire regions of the corresponding $R$ and $Y$ values. They also improve the description of the double dissociation of water defining cut (ii) by the CR-CCSD(T) method in the $R=R_{e}-2.5 R_{e}$ region. For example, in the case of cut (i), the CR-CC(2,3),A approach reduces the $65,167,698$, 1,811 , and $1,699 \mathrm{~cm}^{-1}$ errors in the CR-CCSD(T)/X $=4$ results, relative to the corresponding $\operatorname{MRCI}(\mathrm{Q}) / X=4$ data, at $R=1.25 R_{e}, 1.5 R_{e}, 2 R_{e}, 3 R_{e}$, and $5 R_{e}$ to $35,97,483$, 1,213 , and $954 \mathrm{~cm}^{-1}$, respectively. With an exception of $R=1.25 R_{e}$ and $1.5 R_{e}$, where errors remain almost unchanged, the full CR-CC $(2,3)$ method improves the agreement with the $\mathrm{MRCI}(\mathrm{Q}) / X=4$ energies even further, reducing the $483,1,213$, and $954 \mathrm{~cm}^{-1}$ errors in the CR-CC(2,3),A energies, relative to $\mathrm{MRCI}(\mathrm{Q})$, at $R=2 R_{e}, 3 R_{e}$, and $5 R_{e}$ to 351,813 , and $625 \mathrm{~cm}^{-1}$, respectively. We can clearly see the benefits of using the full CR-CC $(2,3)$ approach, as opposed to CR-CC(2,3),A or CCSD $(2)_{T}$. Similar benefits are observed, when we compare the results of the CR-CC $(2,3), \mathrm{A}$ and full CR-CC $(2,3)$ calculations in the $R=R_{e}-2 R_{e}$ region of cut (ii) and for the the entire cut (iii). The $86-1,059 \mathrm{~cm}^{-1}$ errors, relative to $\mathrm{MRCI}(\mathrm{Q})$, in the $\mathrm{CR}-\mathrm{CC}(2,3), \mathrm{A} / X=4$ results obtained in the $R=R_{e}-2 R_{e}$ region of cut (ii), which are obviously smaller than the $154-1,917 \mathrm{~cm}^{-1}$ errors obtained with CR-CCSD(T), reduce to $79-709 \mathrm{~cm}^{-1}$, when the full CR-CC $(2,3) / X=4$ method is employed. The $68-398 \mathrm{~cm}^{-1}$ differences between the $\mathrm{CR}-\mathrm{CC}(2,3), \mathrm{A} / X=4$ and $\operatorname{MRCI}(\mathrm{Q}) / X=4$ energies in the $Y=0.8-1.75 \AA$ region of cut (iii) reduce to $63-321 \mathrm{~cm}^{-1}$, when the full CR-CC $(2,3) /$ 
$X=4$ method is used. The use of other aug-cc-pCVXZ basis sets does not change any of these accuracy patterns in a substantial manner.

We can conclude that the full CR-CC $(2,3)$ approach, with the complete treatment the diagonal matrix elements of the similarity-transformed Hamiltonian of CCSD involving triply excited determinants, provides the overall best results when compared to all other non-iterative triples methods examined in this work. It is true that the CR-CC $(2,3)$ approach fails in the $R>2 R_{e}$ region of cut (ii) and it is also true that the CR-CC $(2,3)$ energies for smaller stretches of the $\mathrm{O}-\mathrm{H}$ bond(s) appear to be somewhat less accurate than the corresponding $\operatorname{CCSD}(\mathrm{T})$ energies. We must remember, however, that one needs quadruply excited clusters in the $R>2 R_{e}$ region of cut (ii), while the perfect agreement between the $\operatorname{CCSD}(\mathrm{T})$ and $\operatorname{MRCI}(\mathrm{Q})$ data at smaller stretches of the $\mathrm{O}-\mathrm{H}$ bond(s) is not necessarily a desired behavior. Indeed, the explicit inclusion of quadruples in $\mathrm{CC}$ calculations, which can be done in the region of smaller stretches of the $\mathrm{O}-\mathrm{H}$ bond(s) via the CCSD(TQ), b approach, makes the agreement between the $\mathrm{CC}$ and $\mathrm{MRCI}(\mathrm{Q})$ data less perfect, as already explained above. These observations agree with the performance of the CR-CC $(2,3)$ approach in a variety of benchmark calculations reported in Refs. [41,42,44], where it has been noted that the $\mathrm{CR}-\mathrm{CC}(2,3)$ energies are very close to the full CCSDT energies, not only when CCSDT works, but also when it fails, as is the case of multiple bond dissociations. In particular, as shown in Refs. [41,44], the full CCSDT approach completely fails in the $R>2 R_{e}$ region of the double dissociation of water, analogous to our cut (ii). Thus, the failure of CR-CC $(2,3)$ in the same region is a desired result, since one cannot break both $\mathrm{O}-\mathrm{H}$ bonds in the water molecule without quadruply excited clusters, and the approximate triples methods, such as CR-CC $(2,3)$, should not be better in this regard than full CCSDT.

In view of the above discussion, it is very important to examine what happens with the $\mathrm{CR}-\mathrm{CC}(2,3)$ energies if we augment them by quadruples, as is done in the CR-CC $(2,3)+Q(a)$ and CR-CC $(2,3)+\mathrm{Q}(\mathrm{b})$ calculations. As shown in Tables 3 to 9 , the overall agreement between the CR-CC $(2,3)+\mathrm{Q}(x)(x=$ $a, b)$ and $\operatorname{MRCI}(\mathrm{Q})$ data is quite remarkable. For example, in the case of cut (i), corresponding to the dissociation of a single $\mathrm{O}-\mathrm{H}$ bond, the $39-351 \mathrm{~cm}^{-1}$ unsigned differences between the CR-CC $(2,3) / X=4$ and $\mathrm{MRCI}(\mathrm{Q}) / X=4$ energies in the $R=R_{e}-2 R_{e}$ region, in which these energies increase to more than $35,000 \mathrm{~cm}^{-1}$, reduce to $2-86 \mathrm{~cm}^{-1}$ when the $\mathrm{CR}-\mathrm{CC}(2,3)+\mathrm{Q}(\mathrm{b}) / X=4$ approach is employed. The CR-CC $(2,3)+Q(a) / X=4$ method provides similar results, although the somewhat more complete (cf. Table 1) CR-CC $(2,3)+Q(b)$ approximation seems better. The maximum error, relative to $\operatorname{MRCI}(\mathrm{Q})$, characterizing the CR-CC $(2,3) / X=4$ calculations in the entire range of $R$ values, of $813 \mathrm{~cm}^{-1}$, reduces in the $\mathrm{CR}-\mathrm{CC}(2,3)+\mathrm{Q}(\mathrm{b}) / X=$
4 calculations to $395 \mathrm{~cm}^{-1}$. Again, the CR-CC $(2,3)+$ $\mathrm{Q}(\mathrm{a}) / X=4$ approach is almost as effective. The use of other aug-cc-pCV $X Z$ basis sets leads to similar error reductions.

The $79-709 \mathrm{~cm}^{-1}$ differences between the CR-CC $(2,3) /$ $X=4$ and $\mathrm{MRCI}(\mathrm{Q}) / X=4$ energies in the $R=R_{e}-2 R_{e}$ region of cut (ii), where both $\mathrm{O}-\mathrm{H}$ bonds are simultaneously stretched and where the energy goes up to more than 67,000 $\mathrm{cm}^{-1}$, reduce (in absolute value) to $24-121 \mathrm{~cm}^{-1}$, when the CR-CC $(2,3)+\mathrm{Q}(\mathrm{a}) / X=4$ method is employed, and 13-115 $\mathrm{cm}^{-1}$, when the CR-CC $(2,3)+\mathrm{Q}(\mathrm{b}) / X=4$ approach is used. The $\mathrm{CR}-\mathrm{CC}(2,3)+\mathrm{Q}(\mathrm{b})$ approach remains quite accurate up to $R=3 R_{e}$, where the energy is already larger than 80,000 $\mathrm{cm}^{-1}$. The difference between the CR-CC $(2,3)+\mathrm{Q}(\mathrm{b}) / X=4$ and $\mathrm{MRCI}(\mathrm{Q}) / X=4$ energies is only slightly larger than $500 \mathrm{~cm}^{-1}$ in the $R=3 R_{e}$ region. The CR-CC $(2,3)+\mathrm{Q}(\mathrm{a})$ approximation shows the signs of unstable behavior in the $R>2 R_{e}$ region, with the signed errors relative to $\mathrm{MRCI}(\mathrm{Q})$ changing from more than $+2,000 \mathrm{~cm}^{-1}$ to $\sim(-3,000)$ $(-2,000) \mathrm{cm}^{-1}$, which is partly due to the fact that we use the first-order MBPT estimates of doubly excited cluster amplitudes in defining the $(\mathrm{Q})$ corrections of $\mathrm{CR}-\mathrm{CC}(2,3)+\mathrm{Q}$ (a) (or CR-CCSD(TQ),a $[11-13,20])$ and partly due to the heuristic nature of the CR-CC $(2,3)+\mathrm{Q}$ approaches, which should eventually be replaced by the more consistent CR-CC $(2,4)$ theory. The somewhat ad hoc nature of the CR-CC $(2,3)+\mathrm{Q}$ approximations may also be responsible, at least in part, for the $\sim 3,000 \mathrm{~cm}^{-1}$ differences between the CR-CC $(2,3)+Q(b)$ and $\mathrm{MRCI}(\mathrm{Q})$ energies in the $R=4 R_{e}-5 R_{e}$ region of cut (ii). Again, the accuracy patterns observed in the CR$\mathrm{CC}(2,3)+\mathrm{Q}(\mathrm{a})$ and $\mathrm{CR}-\mathrm{CC}(2,3)+\mathrm{Q}$ (b) calculations for cut (ii), when compared to $\mathrm{MRCI}(\mathrm{Q})$, are essentially independent of the aug-cc-pCVXZ basis set employed in these calculations.

The CR-CC $(2,3)+Q(a)$ and $C R-C C(2,3)+Q$ (b) results for cut (iii), corresponding to the $C_{2 v}$ dissociation pathway into $\mathrm{H}_{2}\left(X^{1} \Sigma_{g}^{+}\right)$and $\mathrm{O}\left(2 p^{4}{ }^{1} D\right)$, are very good as well. Both $\mathrm{CR}-\mathrm{CC}(2,3)+\mathrm{Q}(\mathrm{a})$ and $\mathrm{CR}-\mathrm{CC}(2,3)+\mathrm{Q}(\mathrm{b})$ methods reduce the errors resulting from the $\mathrm{CR}-\mathrm{CC}(2,3)$ calculations. The overall accuracy of the $\mathrm{CR}-\mathrm{CC}(2,3)+\mathrm{Q}(\mathrm{b})$ approach in the $Y=0.8-1.75 \AA$ region of cut (iii), as judged by the differences with $\operatorname{MRCI}(\mathrm{Q})$, is better than the accuracy of the CCSD(TQ),b method, which performs very well in this case. It is encouraging to see the relatively small and monotonically increasing $21-210 \mathrm{~cm}^{-1}$ differences between the CR$\mathrm{CC}(2,3)+\mathrm{Q}(\mathrm{b}) / X=4$ and $\operatorname{MRCI}(\mathrm{Q}) / X=4$ energies in the $Y=0.8-1.75 \AA$ A region of cut (iii), where energies go up to $\sim 57,000 \mathrm{~cm}^{-1}$ above the minimum.

The overall agreement between the $\mathrm{CR}-\mathrm{CC}(2,3)+\mathrm{Q}(\mathrm{b})$ and $\operatorname{MRCI}(\mathrm{Q})$ results for all three potential surface cuts examined in this study is excellent, particularly if we keep in mind the single-reference nature of the $\mathrm{CR}-\mathrm{CC}(2,3)+\mathrm{Q}(\mathrm{b})$ calculations. The fact that with an exception of the $R=$ $4 R_{e}-5 R_{e}$ region of cut (ii) and the $Y=2.0-4.0 \AA$ region of cut (iii), the $\mathrm{CR}-\mathrm{CC}(2,3)+\mathrm{Q}(\mathrm{b})$ and $\mathrm{MRCI}(\mathrm{Q})$ energies 
agree to within $500 \mathrm{~cm}^{-1}$ and, in most cases, to within 100 $\mathrm{cm}^{-1}$ or less is a clear demonstration of the large potential offered by the CR-CC theories. The CR-CC $(2,3)$ and CR-CC $(2,3)+Q(b)$ methods seem to be at least as effective as the $\operatorname{CCSD}(\mathrm{T})$ and $\operatorname{CCSD}(\mathrm{TQ}), \mathrm{b}$ methods in the vicinity of the equilibrium geometry, where the many-electron correlation effects are primarily of dynamical nature, while providing the accuracy comparable to the $\operatorname{MRCI}(\mathrm{Q})$ approach in the higher-energy potential energy surface regions characterized by large non-dynamical correlation effects. In the case of the water molecule, we seem to be able to obtain the relatively small differences between the results of the $\mathrm{CR}-\mathrm{CC}(2,3)+\mathrm{Q}(\mathrm{b})$ and $\operatorname{MRCI}(\mathrm{Q})$ calculations, on the order of $100-500 \mathrm{~cm}^{-1}$ or less, for the energies as large as $60,000-70,000 \mathrm{~cm}^{-1}$ above the global minimum. This is an encouraging finding, considering the single-reference character and the relatively low cost of all CR-CC calculations (cf. Table 1). This is also very promising from the point of view of applying the CR-CC methods in calculations aimed at the construction of accurate global potential functions for dynamical studies. The proximity of the CR-CC $(2,3)+Q(b)$ and $\mathrm{MRCI}(\mathrm{Q})$ results for the large portion of the global potential energy surface of water and the fact that the $\mathrm{CR}-\mathrm{CC}(2,3)+\mathrm{Q}(\mathrm{b})$ approach remains as accurate as the $\operatorname{CCSD(TQ),b~method~in~the~vicinity~of~the~}$ equilibrium geometry open up new avenues for constructing highly accurate global potentials, since switching between the CR-CC $(2,3)+Q(b)$ and $\mathrm{MRCI}(\mathrm{Q})$ energies, which are so similar, should be quite straightforward. In the future, we will replace the somewhat heuristic $\mathrm{CR}-\mathrm{CC}(2,3)+\mathrm{Q}$ approximations tested in this work by the properly derived CR-CC $(2,4)$ theory, exploiting the general formalism described in Refs. $[41,42]$. The excellent CR-CC $(2,3)+Q$ results for water obtained in this work prompt such a development.

Let us, finally, address the issue of the rather substantial differences between the $\mathrm{MRCI}(\mathrm{Q}) / X=4$ or $\mathrm{MRCI}(\mathrm{Q}) / X=$ 5 and ES potential energy surfaces in the regions of the intermediate $R$ and $Y$ values, and intermediate or higher, but not the highest energies, which are as much as $700-800 \mathrm{~cm}^{-1}$ for cut (i) in the $R \approx 2.5 R_{e}$ region, $600-800 \mathrm{~cm}^{-1}$ for cut (ii) in the $R \approx 1.75 R_{e}$ region, and $3,500-3,600 \mathrm{~cm}^{-1}$ for cut (iii) in the $Y \approx 1.3 \AA$ region. Such differences cannot be explained by the neglect of the relativistic, non-adiabatic, and quantum electrodynamical effects in $\mathrm{MRCI}(\mathrm{Q})$ calculations. They indicate that either the $\mathrm{MRCI}(\mathrm{Q})$ approach employing large basis sets is insufficiently accurate or the ES potential needs further refinement in the above regions. Although both interpretations are possible, we tend to believe that the accuracy of the ES potential function in the regions of intermediate internuclear separations and energies, which connect the spectroscopic and asymptotic regions, is not as high as the accuracy of the ES surface around the minimum, up to about $19,000 \mathrm{~cm}^{-1}$, and in the $\mathrm{H}\left(1 s^{2} S\right)+\mathrm{OH}\left(X^{2} \Pi\right)$, $2 \mathrm{H}\left(1 s^{2} S\right)+\mathrm{O}\left(2 p^{4}{ }^{3} P\right)$, and $\mathrm{H}_{2}\left(X^{1}{ }^{1} \Sigma_{g}^{+}\right)+\mathrm{O}\left(2 p^{4}{ }^{1} D\right)$ asymptotic regions. We base our belief on the close proximity of the MRCI $(\mathrm{Q})$ and $\mathrm{CR}-\mathrm{CC}(2,3)+\mathrm{Q}(\mathrm{b})$ energies in the regions of the intermediate $R$ and $Y$ values, where the $\operatorname{MRCI}(\mathrm{Q}) / X=4$ or $\operatorname{MRCI}(\mathrm{Q}) / X=5$ and ES potentials significantly differ. This is shown in Table 9 , where we compare the differences between the best CR-CC $(2,3)+\mathrm{Q}(\mathrm{b}) / X=$ 4 energies and the corresponding $\operatorname{MRCI}(\mathrm{Q}) / X=4$ data with the differences between the CR-CC $(2,3)+\mathrm{Q}(\mathrm{b}) / X=4$ and ES energies, and the analogous differences between the $\operatorname{MRCI}(\mathrm{Q}) / X=4,5$ and ES energies. As one can see, the $700-800 \mathrm{~cm}^{-1}$ differences between the $\mathrm{MRCI}(\mathrm{Q}) / X=4,5$ and $\mathrm{ES}$ energies in the $R \approx 2.5 R_{e}$ region of cut (i) are very similar to the $\sim 1,000 \mathrm{~cm}^{-1}$ difference between the $\mathrm{CR}-\mathrm{CC}(2,3)+\mathrm{Q}(\mathrm{b}) / X=4$ and $\mathrm{ES}$ energies in the same region. The $600-800 \mathrm{~cm}^{-1}$ differences between the $\operatorname{MRCI}(\mathrm{Q}) / X=$ 4,5 and ES energies in the $R \approx 1.75 R_{e}$ region of cut (ii) are not much different than the $\sim 700 \mathrm{~cm}^{-1}$ difference between the $\mathrm{CR}-\mathrm{CC}(2,3)+\mathrm{Q}(\mathrm{b}) / X=4$ and ES energies in this region. Finally, the $3,500-3,600 \mathrm{~cm}^{-1}$ differences between the $\mathrm{MRCI}(\mathrm{Q}) / X=4,5$ and ES energies in the $Y \approx 1.3 \AA$ region of cut (iii) are very similar to the $3,000-3,500 \mathrm{~cm}^{-1}$ differences between the CR-CC(2,3)+Q(b)/X $=4$ and ES for $Y \approx 1.2-1.3 \AA$. The large consistency between the $\operatorname{MRCI}(\mathrm{Q}) / X=4,5$ and $\mathrm{CR}-\mathrm{CC}(2,3)+\mathrm{Q}(\mathrm{b}) / X=4$ results in the above regions of the potential energy surface of water makes us believe that the energy values provided by both $a b$ initio approaches in these regions are more accurate than those provided by the existing ES potential. This gives us an opportunity to refine the ES global potential in the future by incorporating some MRCI $(\mathrm{Q})$ or $\mathrm{CR}-\mathrm{CC}(2,3)+\mathrm{Q}(\mathrm{b})$ data from the regions of the intermediate $R$ and $Y$ values in the appropriate fitting and energy switching procedures.

\section{Summary and concluding remarks}

In this paper, we have explored an issue of the development of single-reference procedures that could be applied to at least some of the most frequent multi-reference situations, such as single and double bond dissociations, by reporting test calculations for the potential energy surface of the water molecule. We have focused on a few basic renormalized CC methods, including the older CR-CCSD(T), CRCCSD(TQ), a, and CR-CCSD(TQ),b approximations [10-13, $20,29,30]$, and the most recent size extensive CR-CC(2,3),A (or $\operatorname{CCSD}(\mathrm{T})_{T}$ [38]) and full CR-CC $(2,3)$ approaches that belong to a more general class of CR-CC $\left(m_{A}, m_{B}\right)$ schemes $[41,42,44,131]$. In the CR-CCSD(T), CR-CC(2,3),A, and CR-CC $(2,3)$ methods, the relatively inexpensive corrections due to triply excited clusters, similar in the computer cost to the triples corrections of the conventional $\operatorname{CCSD}(\mathrm{T})$ theory, are added to the CCSD energy. In the CR-CCSD(TQ), a and CR-CCSD(TQ),b approaches, the CCSD energy is correc- 
ted for the dominant effects of triply and quadruply excited clusters in a manner reminiscent of the conventional CCSD(TQ) approximations, such as CCSD(TQ),b [20,32] or $\operatorname{CCSD}\left(\mathrm{TQ}_{\mathrm{f}}\right)$ [19]. Since the CR-CC $(2,4)$ approach has not been fully developed and implemented yet, we have considered two approximate forms of the CR-CC $(2,4)$ theory, abbreviated as $\mathrm{CR}-\mathrm{CC}(2,3)+\mathrm{Q}(\mathrm{a})$ and $\mathrm{CR}-\mathrm{CC}(2,3)+\mathrm{Q}(\mathrm{b})$, in which the $\mathrm{CR}-\mathrm{CC}(2,3)$ energies are a posteriori corrected for the effect of quadruply excited clusters by using the information about quadruples extracted from the CR-CCSD(TQ),a or CR-CCSD(TQ),b calculations.

In order to examine the performance and benefits of using the single-reference renormalized CC methods, we have compared the results of the CR-CCSD(T), CR-CCSD(TQ),a, CR-CCSD(TQ), b, CR-CC(2,3),A, CR-CC (2,3), CR-CC (2,3) $+\mathrm{Q}(\mathrm{a})$, and $\mathrm{CR}-\mathrm{CC}(2,3)+\mathrm{Q}(\mathrm{b})$ calculations for the three important cuts of the potential energy surface of the water molecule, including the dissociation of one $\mathrm{O}-\mathrm{H}$ bond, which correlates with the $\mathrm{H}\left(1 s^{2} S\right)+\mathrm{OH}\left(X^{2} \Pi\right)$ asymptote, the simultaneous dissociation of both $\mathrm{O}-\mathrm{H}$ bonds, which leads to the $2 \mathrm{H}\left(1 s^{2} S\right)+\mathrm{O}\left(2 p^{4}{ }^{3} P\right)$ products, and the $C_{2 v^{-}}$-

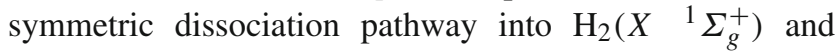
$\mathrm{O}\left(2 p^{4}{ }^{1} D\right)$, with those obtained in the highly accurate MRCI(Q) calculations and those provided by the spectroscopically accurate ES potential function [102]. We have demonstrated that all renormalized CC methods eliminate or considerably reduce the failures of the conventional $\operatorname{CCSD}(\mathrm{T})$ and $\operatorname{CCSD}(\mathrm{TQ}), \mathrm{b}$ approaches in the bond breaking regions of the water potential, while retaining high accuracies of the $\operatorname{CCSD}(\mathrm{T})$ and $\operatorname{CCSD}(\mathrm{TQ}), \mathrm{b}$ methods in the vicinity of the equilibrium geometry. The full CR-CC $(2,3)$ and CR-CC $(2,3)+Q(b)$ methods are particularly effective in this regard. Unlike CCSD $(\mathrm{T})$, the $\mathrm{CR}-\mathrm{CC}(2,3)$ approach provides a faithful description of triply excited clusters $[41,42,44]$, even in the equilibrium region, where the $\operatorname{CCSD}(\mathrm{T})$ approach works well. After correcting the $\mathrm{CR}-\mathrm{CC}(2,3)$ results for quadruples, as is done in the $\mathrm{CR}-\mathrm{CC}(2,3)+\mathrm{Q}(x)(x=\mathrm{a}, \mathrm{b})$ schemes, we obtain the potential energy surfaces of excellent quality, particularly in the $\mathrm{CR}-\mathrm{CC}(2,3)+\mathrm{Q}(\mathrm{b})$ case. Indeed, as shown in this work, the single-reference, RHF-based, CR$\mathrm{CC}(2,3)+\mathrm{Q}(\mathrm{b})$ approach describes the above three cuts of the global potential energy surface of water with accuracies that can only be matched by the high accuracy, CASSCF-based, $\operatorname{MRCI}(\mathrm{Q})$ calculations. We find the small differences between the CR-CC $(2,3)+Q(b)$ and $\operatorname{MRCI}(\mathrm{Q})$ energies, on the order of $100-500 \mathrm{~cm}^{-1}$ or less, for energies as large 60,000 $70,000 \mathrm{~cm}^{-1}$, where the highest possible energies corresponding to the complete atomization of water are on the order of $80,000 \mathrm{~cm}^{-1}$ and where the existing spectroscopically accurate potentials, such as the ES function, can guarantee very high accuracies up to about $19,000 \mathrm{~cm}^{-1}$, to be the most remarkable finding. At the same time, the $\mathrm{CR}-\mathrm{CC}(2,3)+\mathrm{Q}(\mathrm{b})$ approach, which is based on the excellent description of the triply excited cluster contributions by the size extensive CR-CC $(2,3)$ approximation, corrected for the effects of quadruply excited clusters, provides a balanced description of triples and quadruples in the bond breaking and equilibrium regions. In the equilibrium region, the accuracy of the CR$\mathrm{CC}(2,3)+\mathrm{Q}(x)(x=\mathrm{a}, \mathrm{b})$ methods is essentially the same as the accuracy of the CCSD(TQ),b approach, which describes the combined effect of triply and quadruply excited clusters in non-degenerate situations extremely well. Thus, the CR-CC $(2,3)$ method corrected for quadruples enables us to bridge the closed-shell and bond breaking regions of the global potential energy surface of water, while preserving the high accuracy of the CCSD(TQ),b results in the closed-shell regions and matching the high quality of $\mathrm{MRCI}(\mathrm{Q})$ results in regions of stretched chemical bonds, where $\operatorname{CCSD}(\mathrm{TQ}), \mathrm{b}$ (and $\operatorname{CCSD}(\mathrm{T})$, of course) fails.

The excellent agreement between the CR-CC $(2,3)+Q(b)$ and $\mathrm{MRCI}(\mathrm{Q})$ results in regions of intermediate stretches of chemical bonds and higher, but not the highest energies, where the ES surface may be somewhat less accurate, has enabled us to suggest ways of improving the global ES potential function of water that might potentially benefit future reaction dynamics and spectroscopic studies. The regions of intermediate stretches of chemical bonds that connect the spectroscopic and asymptotic regions of the water potential energy surface are not as well understood as the spectroscopic and asymptotic regions. Thus, it is difficult to construct the global potential of water without the high accuracy ab initio data. The large consistency between the CR-CC $(2,3)+Q(b)$ and $\operatorname{MRCI}(\mathrm{Q})$ results in these intermediate regions of the water potential suggest that we should be able to use the CR-CC $(2,3)+Q(b)$ approach and, once developed, the $\mathrm{CR}-\mathrm{CC}(2,4)$ approach to provide the necessary information to improve the ES and other existing global potential functions.

Acknowledgments We dedicate this paper to Professor Mark S. Gordon in celebration of his 65 th birthday. P.P. would like to thank Professor Kim K. Baldridge for inviting him to contribute to a special issue of Theoretical Chemistry Accounts in honor of his best friend and collaborator, Professor Mark S. Gordon, whose seminal contributions to theoretical and computational chemistry, and an exemplary service to the US and international chemistry community, have inspired him over the years. He would also like to thank Universidade de Coimbra, where this study was conceived and carried out, and to the members of the Coimbra Theoretical and Computational Chemistry group, for the hospitality during his sabbatical leave. This work has been supported by the Chemical Sciences, Geosciences and Biosciences Division, Office of Basic Energy Sciences, Office of Science, U.S. Department of Energy (Grant No. DE-FG02-01ER15228; P.P.), the National Science Foundation (Grant No. CHE-0309517; P.P.), and the Fundação para a Ciência e Tecnologia, Portugal (contracts POCI/QUI/60501/2004, POCI/AMB/60261/2004, and REEQ/128/QUI/2005; A.J.C.V.). The calculations reported in this work were performed on the computer 
systems provided by the High Performance Computing Center at Michigan State University.

\section{References}

1. Coester F (1958) Nucl. Phys. 7:421

2. Coester F, Kümmel H (1960) Nucl Phys 17:477

3. Č́ížek J (1966) J Chem Phys 45:4256

4. Čížek J (1969) Adv Chem Phys 14:35

5. Č́ízek J, Paldus J (1971) Int J Quantum Chem 5:359

6. Gauss J (1998) In: Schleyer PvR, Allinger NL, Clark T, Gasteiger J, Kollman PA, Schaefer III HF, Schreiner PR (eds) Encyclopedia of computational chemistry, Vol. 1. Wiley, Chichester pp 615-636

7. Paldus J, Li X (1999) Adv Chem Phys 110:1

8. Crawford TD, Schaefer III HF (2000) Rev Comp Chem 14:33

9. Paldus J (2003) In: Wilson S (ed) Handbook of molecular physics and quantum chemistry, Vol. 2. Wiley, Chichester pp 272-313

10. Piecuch P, Kowalski K (2000) In: Leszczyński J (ed) Computational chemistry: reviews of current trends, Vol. 5. World Scientific, Singapore, pp 1-104

11. Piecuch P, Kowalski K, Pimienta ISO, McGuire MJ (2002) Int Rev Phys Chem 21:527

12. Piecuch P, Kowalski K, Fan P-D, Pimienta ISO (2003) In: Maruani J, Lefebvre R, Brändas E (eds) Progress in Theoretical Chemistry and Physics, Advanced Topics in Theoretical Chemical Physics, Vol. 12. Kluwer, Dordrecht, pp 119-206

13. Piecuch P, Kowalski K, Pimienta ISO, Fan P-D, Lodriguito M, McGuire MJ, Kucharski SA, Kuś T, Musiał M (2004) Theor Chem Acc 112:349

14. Piecuch P, Włoch M, Lodriguito M, Gour JR (2006) In: Julien J-P, Maruani J, Mayou D, Wilson S, Delgado-Barrio G (eds) Progress in Theoretical Chemistry and Physics, Recent Advances in the Theory of Chemical and Physical Systems Vol. 15. Springer, Berlin, pp 45-106

15. Raghavachari K, Trucks GW, Pople JA, Head-Gordon M (1989) Chem Phys Lett 157:479

16. Purvis III GD, Bartlett RJ (1982) J Chem Phys 76:1910

17. Scuseria GE, Scheiner AC, Lee TJ, Rice JE, Schaefer III HF (1987) J Chem Phys 86:2881

18. Piecuch P, Paldus J (1989) Int J Quantum Chem 36:429

19. Kucharski SA, Bartlett RJ (1998) J Chem Phys 108:9221

20. Kowalski K, Piecuch P (2000) J Chem Phys 113:5644

21. Laidig WD, Saxe P, Bartlett RJ (1987) J Chem Phys 86:887

22. Ghose KB, Piecuch P, Adamowicz L (1995) J Chem Phys 103:9331

23. Piecuch P, Špirko V, Kondo AE, Paldus J (1996) J Chem Phys 104:4699

24. Piecuch P, Kucharski SA, Bartlett RJ (1999) J Chem Phys 110:6103

25. Piecuch P, Kucharski SA, Špirko V (1999) J Chem Phys 111:6679

26. Dutta A, Sherrill CD (2003) J Chem Phys 118:1610

27. Abrams ML, Sherrill CD (2004) J Chem Phys 121:9211

28. Sherrill CD, Piecuch P (2005) J Chem Phys 122:124104

29. Piecuch P, Kowalski K, Pimienta ISO, Kucharski SA (2002) In: Hoffmann MR, Dyall KG (eds) Low-lying potential energy surfaces, ACS Symposium Series, Vol. 828. American Chemican Society, Washington pp 31-64

30. Kowalski K, Piecuch P (2000) J Chem Phys 113:18

31. Kowalski K, Piecuch P (2001) Chem Phys Lett 344:165

32. Piecuch P, Kucharski SA, Kowalski K (2001) Chem Phys Lett 344:176

33. Piecuch P, Kucharski SA, Špirko V, Kowalski K (2001) J Chem Phys 115:5796
34. McGuire MJ, Kowalski K, Piecuch P (2002) J Chem Phys 117:3617

35. Piecuch P, Kowalski K, Pimienta ISO (2002) Int J Mol Sci 3:475

36. Pimienta ISO, Kowalski K, Piecuch P (2003) J Chem Phys 119:2951

37. McGuire MJ, Kowalski K, Piecuch P, Kucharski SA, Musiał M (2004) J Phys Chem A 108:8878

38. Hirata S, Fan P-D, Auer AA, Nooijen M, Piecuch P (2004) J Chem Phys 121:12197

39. Fan P-D, Kowalski K, Piecuch P (2005) Mol Phys 103:2191

40. Kowalski K, Piecuch P (2005) J Chem Phys 122:074107

41. Piecuch P, Włoch M (2005) J Chem Phys 123:224105

42. Piecuch P, Włoch M, Gour JR, Kinal A (2006) Chem Phys Lett 418:467

43. Piecuch P, Hirata S, Kowalski K, Fan P-D, Windus TL (2006) Int J Quantum Chem 106:79

44. Włoch M, Lodriguito MD, Piecuch P, Gour JR (2006) Mol Phys 104:2149

45. Lodriguito MD, Kowalski K, Włoch M, Piecuch P (2006) J Mol Struct:THEOCHEM 771:89

46. Özkan I, Kinal A, Balci M (2004) J Phys Chem A 108:507

47. McGuire MJ, Piecuch P (2005) J Am Chem Soc 127:2608

48. Kinal A, Piecuch P (2006) J Phys Chem A 110:367

49. Cramer CJ, Włoch M, Piecuch P, Puzzarini C, Gagliardi L (2006) J Phys Chem A 110:1991

50. Cramer CJ, Kinal A, Włoch M, Piecuch P, Gagliardi L (2006) J Phys Chem A 110:11557

51. Kinal A, Piecuch P (2007) J Phys Chem A 111:734

52. Gwaltney SR, Head-Gordon M (2000) Chem Phys Lett 323:21

53. Gwaltney SR, Sherrill CD, Head-Gordon M, Krylov AI (2000) J Chem Phys 113:3548

54. Gwaltney SR, Head-Gordon M (2001) J Chem Phys 115:2014

55. Gwaltney SR, Byrd EFC, Van Voorhis T, Head-Gordon M (2002) Chem Phys Lett 353:359

56. Head-Gordon M, Van Voorhis T, Gwaltney SR, Byrd EFC (2002) In: Hoffmann MR, Dyall KG (eds) Low-lying potential energy surfaces, ACS Symposium Series, Vol. 828. American Chemican Society, Washington pp 93-108

57. Musiał M, Bartlett RJ (2005) J Chem Phys 122:224102

58. Schmidt MW, Gordon MS (1998) Annu Rev Phys Chem 49:233

59. Chaudhuri RK, Freed KF, Hose G, Piecuch P, Kowalski K, Włoch M, Chattopadhyay S, Mukherjee D, Rolik Z, Szabados Á, Tóth G, Surján PR (2005) J Chem Phys 122:134105

60. Roos BO, Linse P, Siegbahn PEM, Blomberg MRA (1982) Chem Phys 66:197

61. Andersson K, Malmqvist P-Å, Roos BO, Sadlej AJ, Woliński K (1990) J Phys Chem 94:5483

62. Andersson K, Malmqvist P-Å, Roos BO (1992) J Chem Phys 96:1218

63. Andersson K, Roos BO (1995) In: Yarkony DR (ed) Modern electronic structure theory, Vol. 2. of Advanced Series in Physical Chemistry. World Scientific, Singapore, pp 55-109

64. Rode MF, Werner H-J (2005) Theor Chem Acc 114:309

65. Werner H-J, Knowles PJ (1988) J Chem Phys 89:5803

66. Knowles PJ, Werner H-J (1988) Chem Phys Lett 145:514

67. Abrams ML, Sherrill CD (2003) J Phys Chem A 107:5611

68. Sears JS, Sherrill CD (2005) Mol Phys 103:803

69. Stanton JF (1997) Chem Phys Lett 281:130

70. Hirata S, Nooijen M, Grabowski I, Bartlett RJ (2001) J Chem Phys 114:3919; 115, 3967 (2001) (Erratum)

71. Krylov AI (2001) Chem Phys Lett 338:375

72. Krylov AI, Sherrill CD (2002) J Chem Phys 116:3194

73. Slipchenko LV, Krylov AI (2002) J Chem Phys 117:4694

74. Van Voorhis T, Head-Gordon M (2000) Chem Phys Lett 330:585

75. Byrd EFC, Van Voorhis T, Head-Gordon M (2002) J Phys Chem B 106:8070 
76. Fan P-D, Piecuch P (2006) Adv Quantum Chem 51:1

77. Arponen JS (1983) Ann Phys 151:311

78. Arponen JS, Bishop RF, Pajanne E (1987) Phys Rev A 36:2519

79. Bishop RF, Arponen JS (1990) Int J Quantum Chem Symp 24:197

80. Arponen JS, Bishop RF (1991) Ann Phys 207:171

81. Bishop RF (1991) Theor Chim Acta 80:95

82. Arponen J (1997) Phys Rev A 55:2686

83. Arponen JS, Bishop RF, Pajanne E (1987) Phys Rev A 36:2539

84. Piecuch P, Bartlett RJ (1999) Adv Quantum Chem 34:295

85. Kowalski K, Piecuch P (2001) J Chem Phys 115:2966

86. Kowalski K, Piecuch P (2002) J Chem Phys 116:7411

87. Kowalski K, Piecuch P (2004) J Chem Phys 120:1715

88. Włoch M, Gour JR, Kowalski K, Piecuch P (2005) J Chem Phys 122:214107

89. Piecuch P, Kucharski SA, Kowalski K, Musiał M (2002) Comp Phys Commun 149:71

90. Schmidt MW, Baldridge KK, Boatz JA, Elbert ST, Gordon MS, Jensen JH, Koseki S, Matsunaga N, Nguyen KA, Su SJ, Windus TL, Dupuis M, Montgomery JA (1993) J Comput Chem 14:1347

91. Chipman DM (2006) J Chem Phys 124:044305

92. Li X, Paldus J (2006) J Chem Phys 125:164107

93. Sarker MIM, Kim CS, Choi CH (2005) Chem Phys Lett 411:297

94. Avramov PV, Adamovic I, Ho KM, Wang CZ, Lu WC, Gordon MS (2005) J Phys Chem A 109:6294

95. Kowalski K (2005) J Chem Phys 123:014102

96. Noga J, Bartlett RJ (1987) J Chem Phys 86:7041 ; 89, 3401 (1988) (Erratum)

97. Scuseria GE, Schaefer III HF (1988) Chem Phys Lett 152:382

98. Dunning Jr TH (1989) J Chem Phys 90:1007

99. Woon DE, Dunning Jr TH (1995) J Chem Phys 103:4572

100. Kendall RA, Dunning Jr TH, Harrison RJ (1992) J Chem Phys 96:6769

101. Basis sets were obtained from the Extensible Computational Chemistry Environment Basis Set Database, Version 02/02/06, as developed and distributed by the Molecular Science Computing Facility, Environmental and Molecular Sciences Laboratory which is part of the Pacific Northwest Laboratory, P.O. Box 999, Richland, Washington 99352, USA, and funded by the U.S. Department of Energy. The Pacific Northwest Laboratory is a multi-program laboratory operated by Battelle Memorial Institute for the U.S. Department of Energy under contract DE-AC0676RLO 1830. Contact Karen Schuchardt for further information

102. Varandas AJC (1996) J Chem Phys 105:3524

103. Wallace L, Bernath P, Livingstone W, Hinkle K, Busler J, Gour B, Zhang K (1995) Science 268:1155

104. Oka T (1997) Science 277:328

105. Wayne RP (2000) Chemistry of Atmospheres. Oxford University Press, Oxford, pp 50-58

106. Császár AG, Tarczay G, Leininger ML, Polyansky OL, Tennyson J, Allen WD (2001) In: Demaison J, Sarka K (eds) Spectroscopy from Space, NATO ASI Series C. Kluwer, Dordrecht, pp 317-339
107. Tolchenov RN, Tennyson J, Brault JW, Canas AAD, Schermaul R (2002) J Mol Spectrosc 215:269

108. Tolchenov RN, Tennyson J, Shirin SV, Zobov NF, Polyansky OL, Maurellis AN (2003) J Mol Spectrosc 221:99

109. Polyansky OL, Csaszar AG, Shirin SV, Zobov NF, Bartletta P, Tennyson J, Schwenke DW, Knowles PJ (2003) Science 299:539

110. Tolchenov RN, Naumenko O, Zobov NF, Shirin SV, Polyansky OL, Tennyson J, Carleer M, Coheur P-F, Fally S, Jenouvrier A, Vandaele AC (2005) J Mol Spectrosc 233:68

111. Coheur PF, Bernath PF, Carleer M, Colin R, Polyansky OL, Zobov NF, Shirin SV, Barber RJ, Tennyson J (2005) J Chem Phys 122:074307

112. Tolchenov RN, Tennyson J (2005) J Mol Spectrosc 231:23

113. Varandas AJC, Voronin AI, Riganelli A, Caridade PJSB (1997) Chem Phys Lett 278:325

114. Varandas AJC, Voronin AI, Caridade PJSB, Riganelli A (2000) Chem Phys Lett 331:331

115. Murrell JN, Carter S (1984) J Phys Chem 88:4887

116. Polyansky OL, Jensen P, Tennyson J (1994) J Chem Phys 101:7651

117. Partridge H, Schwenke DW (1997) J Chem Phys 106:4618

118. Varandas AJC (1997) J Chem Phys 107:867

119. Varandas AJC, Voronin AI, Caridade PJSB (1998) J Chem Phys 108:7623

120. Murrell JN, Carter S, Farantos SC, Huxley P, Varandas AJC (1984) Molecular potential energy functions. Wiley, Chichester

121. Varandas AJC (1988) Adv Chem Phys 74:255

122. Varandas AJC (1992) Chem Phys Lett 194:333

123. Varandas AJC, Voronin AI (1995) Mol Phys 85:497

124. Varandas AJC (2004) In: Domcke W, Yarkony DR, Köppel H (eds) Conical Intersections: Electronic Structure, Dynamics, and Spectroscopy, Vol. 15 of Advanced Series in Physical Chemistry. World Scientific, Singapore, pp 205-270

125. Valeev EF, Allen WD, Schaefer III HF, Császár AG (2001) J Chem Phys 114:2875

126. MOLPRO, version 2002.6; a package of ab initio programs; H.-J. Werner, Knowles PJ, Lindh R, Schütz M, Celani P, Korona T, Manby FR, Rauhut G, Amos RD, Bernhardsson A, Berning A, Cooper DL, Deegan MJO, Dobbyn AJ, Eckert F, Hampel C, Hetzer G, Lloyd AW, McNicholas SJ, Meyer W, Mura ME, Nicklass A, Palmieri P, Pitzer R, Schumann U, Stoll H, Stone AJ, Tarroni R, and Thorsteinsson T; see http://www.molpro.net (Birmingham, UK, 2003)

127. DeKock RL, McGuire MJ, Piecuch P, Allen WD, Schaefer III HF, Kowalski K, Kucharski SA, Musiał M, Bonner AR, Spronk SA, Lawson DB, Laursen SL (2004) J Phys Chem A 108:2893

128. Kucharski SA, Bartlett RJ (1999) J Chem Phys 110:8233

129. Kucharski SA, Bartlett RJ (1999) Chem Phys Lett 302:295

130. Nangia S, Truhlar DG, McGuire MJ, Piecuch P (2005) J Phys Chem A 109:11643

131. Piecuch P, Włoch M, Gour JR, Lodriguito MD unpublished 